\title{
Airframe Noise Prediction by Acoustic Analogy: Revisited
}

\author{
F. Farassat and Jay H. Casper \\ NASA Langley Research Center, Hampton, Virginia \\ A. Tinetti \\ NSC Information Systems, Inc., Senior Principal Software Engineer \\ Senior Member AIAA \\ M. H. Dunn \\ National Institute of Aerospace, Consultant \\ Member AIAA
}

\begin{abstract}
AIAA--2006-2564, Presented at the 12th AIAA/CEAS Aeroacoustics Conference, 8-10 May 2006, Cambridge, Massachusetts
\end{abstract}

\begin{abstract}
The present work follows a recent survey of airframe noise prediction methodologies. In that survey, Lighthill's acoustic analogy was identified as the most prominent analytical basis for current approaches to airframe noise research. Within this approach, a problem is typically modeled with the Ffowcs Williams and Hawkings (FW-H) equation, for which a geometryindependent solution is obtained by means of the use of the free-space Green function (FSGF). Nonetheless, the aeroacoustic literature would suggest some interest in the use of "tailored" or exact Green's function (EGF) for aerodynamic noise problems involving solid boundaries, in particular, for trailing edge (TE) noise. A study of possible applications of EGF for prediction of broadband noise from turbulent flow over an airfoil surface and the TE is, therefore, the primary topic of the present work. Typically, the applications of EGF in the literature have been limited to TE noise prediction at low Mach numbers assuming that the normal derivative of the pressure vanishes on the airfoil surface. To extend the application of EGF to higher Mach numbers, the uniqueness of the solution of the wave equation when either the Dirichlet or the Neumann boundary condition (BC) is specified on a deformable surface in motion. The solution of Lighthill's equation with either the Dirichlet or the Neumann BC is given for such a surface using EGFs. These solutions involve both surface and volume integrals just like the solution of FW-H equation using FSGF. Insight drawn from this analysis is evoked to discuss the potential application of EGF to broadband noise prediction. It appears that the use of a EGF offers distinct advantages for predicting TE noise of an airfoil when the normal pressure gradient vanishes on the airfoil surface. It is argued that such an approach may also apply to an airfoil in motion. However, for the prediction of broadband noise not directly associated with a trailing edge, the use of EGF does not appear to offer any advantages over the use of FSGF at the present stage of development. It is suggested here that the applications of EGF for airframe noise analysis be continued. As an example pertinent to airframe noise prediction, the Fast Scattering Code of NASA Langley is utilized to obtain the EGF numerically on the surface of a three dimensional wing with a flap and leading edge slat in uniform rectilinear motion. The interpretation and use of these numerical Green functions are then discussed.
\end{abstract}




\section{Introduction}

In the present work we continue the study of airframe noise (AFN) prediction methodologies ${ }^{1}$. This investigation is motivated by the need to develop physics-based AFN prediction tools. As future airport-noise abatement goals become more stringent, it will eventually become imperative to predict overall noise levels during an aircraft's design stage, including its airframe design. The success of such a "quiet-design" approach will depend on a physics-based prediction methodology, i.e., a methodology in which the noise prediction process incorporates modeling that is derived from first principles. Noise prediction tools that include as much physics-based modeling as possible are highly tractable and robust, and thereby well suited for future design guidance.

The current AFN methodology study began with a survey of the prediction methods that are currently in use or in various stages of development ${ }^{1}$. In that work, present approaches to AFN prediction are categorized into four groups, three of which are referred to as "semi-empirical," "fully analytic," and "fully numerical." In the fourth approach, a computational fluid dynamics (CFD) simulation provides the source description, and the radiated sound is predicted by an appropriate acoustic formulation based on Lighthill's acoustic analogy ${ }^{2}$. At present, only the CFD/acoustic-analogy (CFD/AA) approach provides the potential to develop the necessary physics-based prediction tools within the required time frame ${ }^{1}$. As this conclusion has remained relevant for the past decade, NASA's development of a physics-based AFN methodology has been largely concentrated within the CFD/AA approach.

The advantages of a CFD/AA methodology result from the very notion of "acoustic analogy," itself. Morris and Farassat describe an acoustic analogy as "any aerodynamic noise theory in which the equations of motion for a compressible fluid are rearranged in a way that separates linear acoustic propagation effects." 3 Furthermore, "this rearrangement results in a set of equivalent sources that are assumed to be non-negligible in a limited region of space." Therefore, within the CFD/AA approach, the CFD solution is regarded as input data to a stand-alone acoustic propagation algorithm. As such, the propagated sound can be predicted to observers in arbitrary locations, e.g., at distances many times larger than the spatial domain of the input CFD solution. Moreover, such an acoustic-analogy based algorithm is a direct application of an explicit, analytical formulation for radiated sound. Thus, there is no additional error incurred during the acoustic calculation; i.e., the farfield noise prediction is as accurate as the computed source input.

The most commonly used governing equation for AFN prediction is the Ffowcs Williams-Hawkings $(\mathrm{FW}-\mathrm{H})$ equation ${ }^{4}$. The solution of this equation is obtained by using the free-space Green's function (FSGF) which is independent of the aircraft geometry. The usefulness of this approach has been clearly established by the prevalence of noise prediction tools based on solution of FW-H equation. Nonetheless, in recent years a number of technical papers and books have appeared that promote the use of the so-called tailored or exact Green's function as an analytical tool for AFN problems $5-12$. Although Howe has been the leading advocate of the use of EGF, the present authors believe that it was Goldstein who first suggested the use of EGF in an important paper in $1974^{13}$ and in his book on aeroacoustics ${ }^{14}$. In Goldstein's works, the EGF was discussed in connection with the possible choices of the Green's function in acoustic problems that are solved in the presence of solid bodies.

We feel that it is time that the use of EGF approach be reviewed carefully and compared to the use of the FSGF in AFN prediction problems. Such a review and comparison are presented in this paper. Our approach is as follows. In Section 2 , we briefly discuss the history of broadband noise prediction problem, what is known from previous research, and why the use of EGF has attracted so much attention from aeroacousticians. Then we explicitly state the mathematical problem that we address here which is whether EGF approach can be used for a surface in motion. We accomplish this by proving some uniqueness theorems for the wave equation. To this end, in Section 3 we prove rigorously that the solution of the initialboundary value problem of the wave equation is unique with either the Dirichlet or the Neumann boundary condition specified on a deformable body in motion. This means that the EGF may be found for a moving surface and used for AFN prediction. In Section 4, we discuss the implications of this result when used for broadband noise prediction. Our main conclusion is that there seems to be clear advantages of using EGF over the FSGF for TE noise prediction at low Mach numbers and perhaps even at high Mach numbers. It is recommended that the use of EGF be explored further in AFN prediction. Finally, in Section 5 we give examples of EGF for the nontrivial case of two three dimensional wings one of which has a trailing edge flap and a leading edge slat. These EGF are constructed numerically for a fixed source frequency on the wing surface for several observer positions. Concluding remarks follow in Section 6. 


\section{The Airframe Broadband Noise Problem}

\subsection{Some History}

Broadband noise radiation from airfoil surfaces has been studied in connection with aircraft and underwater acoustics by

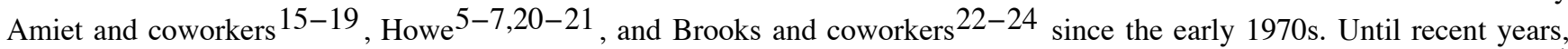
almost all of noise prediction methodologies depended on the use of Ffowcs Williams and Hawkings (FW-H) equation 4 whose solution uses the FSGF of linear wave equation. The use of FSGF allows various closed form solutions of FW-H equation because of extreme simplicity of the analytic form of FSGF and its easily visualizable geometry, e.g., the collapsing sphere visualization of the characteristic cone of the wave equation in four dimensions. Two fundamental papers by Ffowcs Williams and Hall ${ }^{25}$ and Crighton and Lippington ${ }^{26}$ have contributed greatly in understanding the physics of TE noise generation by quadrupoles and vortices passing in the vicinity of the edge. The results of these authors have been verified by many others 27 .

In recent years researchers within NASA, the universities, aircraft and engine industry in the U.S., as well as similar organizations in Europe and Asia have conducted intensive research on AFN. Many technical papers have been published. There has been a sudden surge of interest in the use of EGF as evidenced by the number of authors who are using EGF in their publications. The chief advocate of the use of EGF in recent years is Howe ${ }^{5,6}$. But Goldstein had already suggested the use of EGF in a paper in $1974^{13}$ and in his well-known book aeroacoustics ${ }^{14}$ which is based on an earlier NASA publication with the same name ${ }^{28}$. It is also true that the authors of the two fundamental papers on TE noise 25,26 used the EGF for the two dimensional problems that they considered. We will discuss below some of the advantages of the use of EGF in AFN prediction.

An open and important question that the researchers who propose the use of EGF have not addressed involves the uniqueness of the solution of the wave equation with the Dirichlet or Neumann boundary condition (BC) on a moving surface. Apparently, the prevailing view is that because of the slow speed of aircraft during take-off and landing (about Mach 0.2), the uniqueness theorem for a stationary surface will apply in AFN predictions. Although, intuitively, the solution's uniqueness appears obvious, it is not enough to rely on intuition alone. If the uniqueness theorem breaks down for a moving surface, much of the recent results based on EGF will be in doubt. We, therefore, propose to prove here two uniqueness theorems for the wave equation with the Dirichlet or the Neumann BC for a moving surface. These two theorems constitute the main contributions of the present paper. We will then discuss the implications of these results as used in broadband noise prediction.

\subsection{What We Know Now}

We give a summary of what is known in predicting broadband noise caused by turbulent flow over an airfoil and the TE noise. Let us start with the acoustic analogy using the FW-H equation whose solution requires FSGF. The FW-H equation as originally proposed by Ffowcs William and Hawkings is ${ }^{4}$ :

$$
\square^{2} p^{\prime}=\frac{\partial}{\partial t}\left[\rho_{0} v_{n} \delta(f)\right]-\frac{\partial}{\partial x_{i}}\left[p n_{i} \delta(f)\right]+\frac{\partial^{2}}{\partial x_{i} \partial x_{j}}\left[H(f) T_{i j}\right]
$$

Here, the moving surface is described by $f(\boldsymbol{x}, t)=0$ such that $\nabla \mathbf{f}=\boldsymbol{n}, \boldsymbol{n}$ is the unit outward normal. This assumption implies that $f>0$ outside the moving surface (see fig. 1). Also $p^{\prime}=c^{2} \rho^{\prime}=c^{2}\left(\rho-\rho_{0}\right), c$ and $\rho_{0}$ are the speed of sound and density in the undisturbed medium, respectively, $v_{n}, p$ and $T_{i j}$ are the local normal velocity of the surface, the local gage pressure of the surface, and the Lighthill stress tensor, respectively. The Heaviside and the Dirac delta functions are denoted $H(f)$ and $\delta(f)$, respectively.

Ffowcs Williams later proposed to use a penetrable (porous or permeable) data surface to account for nonlinearities in the vicinity of a moving surface ${ }^{29}$. We again assume that the penetrable surface defined by $f(\boldsymbol{x}, t)=0$ and the fluid velocity is denoted by $\boldsymbol{u}$. The FW-H equation for penetrable data surface, FW $-H_{\mathrm{pds}}$, is 29,30 : 


$$
\square^{2} c_{0}^{2} \rho^{\prime} \equiv \square^{2} p^{\prime}=\frac{\partial}{\partial t}\left[\rho_{0} U_{n} \delta(f)\right]-\frac{\partial}{\partial x_{i}}\left[L_{i} \delta(f)\right]+\frac{\partial^{2}}{\partial x_{i} \partial x_{j}}\left[T_{i j} H(f)\right]
$$

We have used the following notations in the above equation:

$$
\begin{aligned}
& U_{n}=\left(1-\frac{\rho}{\rho_{0}}\right) v_{n}+\frac{\rho u_{n}}{\rho_{0}} \\
& L_{i}=p \delta_{i j} n_{j}+\rho u_{i}\left(u_{n}-v_{n}\right)
\end{aligned}
$$

where $\delta_{i j}$ is the Kronecker delta. The philosophy behind using $\mathrm{FW}-\mathrm{H}_{\mathrm{pds}}$ is to locate the data surface $f=0$ to enclose a moving noise surface, in such a way that all quadrupoles producing non-negligible noise are included within this surface, so that no volume integration of the quadrupoles outside the data surface is necessary. This data surface must be located in the region of high grid density in order to adequately account for the upper spectrum of a broadband AFN calculation. For this reason, one would like this surface to be as small as possible, because of the computer intensive nature of turbulence simulation $^{31-36}$.

The formal solution of eqs.(1) and (2) has two surface integrals involving local normal velocity and surface pressure and a volume integral of the Lighthill quadrupole ${ }^{4,30}$. This solution form is a consequence of the fact that we have used FSGF in the derivation. For studying broadband noise some sort of statistical analysis of the solution must be performed as, for example, is done by Morris and Farassat for jet noise analysis ${ }^{3}$ to relate the statistics of the noise to the statistics of turbulent flow. When both surface and volume integrals appear in the solution, the algebra for statistical analysis becomes very complicated.

Concerning the surface data needed for noise calculations, it is clear that, because of the appearance of the Dirac delta functions in eqs. (1) and (2), no normal derivative of surface pressure should appear in the solution. This is expected because of the following result from generalized function theory 37,38 :

$$
p(\boldsymbol{x}, t) \delta(f)=[p(\boldsymbol{x}, t)]_{f=0} \delta(f) \Rightarrow \nabla[p(\boldsymbol{x}, t)]_{f=0}=\nabla_{T} p(\boldsymbol{x}, t)
$$

where $\nabla_{T} p(\boldsymbol{x}, t)$ is the gradient of $p(\boldsymbol{x}, t)$ tangent to the surface. Therefore, the surface input data used for source description for FW-H equation is of Dirichlet type. It is important to remember this when we discuss the uniqueness theorems for the solution of wave equation with the Dirichlet or the Neumann BC.

Some important fact to know about the use of FW-H and equations for broadband noise prediction are:

1- These equations are exact and given the exact input data that accurately models the source, one should get the correct radiated noise 27 ,

2- The surface pressure $p$ may include an acoustic component which should be included in predicting noise off the surfa$\mathrm{ce}^{4,27}$ (see next subsection),

3 - There is the possibility of noise cancellation from surface and volume sources in eqs.(1) ${ }^{39}$. This phenomenon is direction and frequency dependent.

4- Doing statistical analysis on solutions based on FW-H and FW - $H_{\mathrm{pds}}$ is difficult if all the integral terms in the solution are retained. Relating the statistics of the broadband noise to the statistics of the volume and surface sources is essential in understanding and reduction of AFN.

It is important to recognize that, in general, the volume sources involved in the generation of broadband noise occupy small region of space in the vicinity of the surface but can have very high velocity gradients. Conceptually, a compressible turbulence simulation would model the acoustic waves propagating over the surface. The problem is that compressible turbulence simulation is computer intensive and thus expensive and we prefer incompressible turbulence simulation. However, at present, compressible turbulence simulations at meaningful scales are prohibitively expensive, and therefore research interests are pursued in the area of incompressible simulations. The question is whether surface integration of the acoustic pressure can be avoided if at all possible. The affirmative answer for some noise prediction problems is given in the next Subsection. 
The possible cancellation between volume and surface integrals is another matter. In general, this problem should not exist if the penetrable data surface for eq. (2) encloses the entire region of non-negligible turbulence intensity and vortical flow. This is not always possible because of the high cost of turbulent flow simulation. Although a limited forms of solutions of FW-H equation employing the FSGF have been tested so far, the possibility of adding some of the surface terms hidden in the volume source term of eqs.(1) and (2) to the loading term as discussed by Farassat, Myers and Brentner ${ }^{40,41}$ to avoid this problem has not been explored. Also, improvements in flow simulation to extend the region of computation for use in a solution based on FSGF in FW $-\mathrm{H}_{\mathrm{pds}}$ will alleviate the cancellation problem. However, the issue of specifying statistics on the data surface, based on the flow simulation, remains. The use of EGF can avoid this difficulty in statistical formulation in certain situations, e.g., TE noise prediction, but not the need for an accurate turbulence simulation.

\subsection{Why Use EGF?}

Howe $^{7}$ discusses the Curle problem ${ }^{42}$ and the solution that Powell gives using the image of quadrupoles reflected in the plane boundary 43 . Howe points out that to predict the noise using the Curle formula, one has to integrate the acoustic pressure over a large portion of the plane boundary. In the Powell formulation, this surface integral is simply equal to the contribution of the image quadrupoles. Howe considers Powell's solution which is based on the EGF more appropriate. We will give another example which is the piston in the wall problem.

Figure 2 shows a piston in an infinite baffle with normal velocity distribution $v_{n}\left(x_{1}, x_{2}, t\right)$ on the piston surface $\Omega_{S}$. The solutions of this problem by using FSGF in FW-H equation, and by Rayleigh using two different EGF based on the Dirichlet and Neumann BCs are ${ }^{44}$ :

$$
\begin{aligned}
& 4 \pi p^{\prime}(\boldsymbol{x}, t)=\int_{\Omega_{s}} \frac{\left[\rho_{0} \dot{v}_{n}\right]_{\mathrm{ret}}}{r} d S+\int_{\mathbb{R}^{2}} \frac{[\dot{p}]_{\mathrm{ret}} \cos \theta}{c r} \mathrm{dS}+\int_{\mathbb{R}^{2}} \frac{[p]_{\mathrm{ret}} \cos \theta}{r^{2}} \mathrm{dS} \quad(\mathrm{FW}-H \text { eq. }) \\
& 2 \pi p^{\prime}(\boldsymbol{x}, t)=\int_{\mathbb{R}^{2}} \frac{[\dot{p}]_{\mathrm{ret}} \cos \theta}{c r} \mathrm{dS}+\int_{\mathbb{R}^{2}} \frac{[p]_{\mathrm{ret}} \cos \theta}{r^{2}} \mathrm{dS} \quad(\text { Dirichlet BC) } \\
& 2 \pi p^{\prime}(\boldsymbol{x}, t)=\int_{\Omega_{s}} \frac{\left[\rho_{0} \dot{v}_{n}\right]_{\mathrm{ret}}}{r} d S=-\int_{\Omega_{s}} \frac{[\partial p / \partial n]_{\mathrm{ret}}}{r} d S \quad \text { (Neumann BC) }
\end{aligned}
$$

It is clear that the solutions given by eqs.(6) and (7) which require the knowledge of the surface pressure over the entire two dimensional space, have basically two shortcomings as compared to that given by eq.(8). First more input data are needed to get the acoustic pressure and, second, the prediction is more costly. Note that the simplest solution, eq.(8), uses the Neumann $\mathrm{BC}$.

Let us now discuss why Howe recommends the use of the EGF with the Neumann BC $\partial p / \partial n=0$ for the TE noise problem when the airfoil moves at low Mach number ${ }^{7}$. Let the typical wavelength of the sound associated with the vortical flow over the TE be much greater than the chord. Then one can use incompressible turbulence simulation near the TE and use the volume quadrupoles as the source of sound which will be scattered by the TE. The use of the Neumann BC eliminates the need for the knowledge of the pressure on the surface. This type of $\mathrm{BC}$ means that $\partial p / \partial n$ is specified on the airfoil surface which for a stationary surface is $\partial p / \partial n=0$. The condition imposed on the EGF in this case is that $\partial G / \partial n=0$. The only sources remaining are the Lighthill quadrupoles which are volume sources. An additional advantage here is that by having only one integral to evaluate the radiated noise, statistical analysis of the acoustic pressure based on the statistics of the turbulent flow is considerably simplified. Even for the case of high frequency TE noise where the typical wavelength of sound is much smaller than the chord, or when the airfoil is in motion, the assumption of $\partial p / \partial n=0$ appears to be acceptable on physical grounds. The turbulent flow simulation near the TE may have to be compressible, but limited to the TE region, and the noncompactness of the volume sources may have to be taken into account for noise calculations.

To study the use of EGF for surfaces in motion, we will next define the problem that we will address in the next section. 


\subsection{Problem Definition}

The broadband airframe noise problem that we will address here is due to the turbulent flow in the boundary layer (BL) and the passage of the vortical motion in the BL over the TE of a wing and its control surfaces. As is well-known, these are not the only sources of broadband noise of airframe ${ }^{1}$. The turbulent pressure fluctuations on the wing surface and the scattering of the energy in the vortical flow over the TE generate broadband noise that we are concerned with in this paper. Theoretically, the quadrupoles in the BL and the wake of the wing and control surfaces as well as in the flow over the side edges of flaps and slats also generate broadband noise. We are interested in proposing a suitable formulations to predict this broadband noise assuming that high-resolution turbulent simulation data are available to us. In particular, the use of an EGF formulation for this problem will be studied.

\section{Some Uniqueness Theorems for the Initial-Boundary Value Problem of the Wave Equation}

The problem that we are considering here is described as follows. Let an arbitrary deformable surface moving in the unbounded three dimensional space $\mathbb{R}^{3}$ be given implicitly by $f(\boldsymbol{x}, t)=0$ such that $\nabla f=\boldsymbol{n}$, where $\boldsymbol{n}$ is the local outward pointing unit normal to the surface (see fig. 3). The symbol $\Omega_{t}$ stands for the region exterior to the surface $f=0$, and $\partial \Omega_{t}$ refers to the boundary of $\Omega_{t}$ described by the equation $f=0$. The fact that $\boldsymbol{n}$ points into the exterior of $f=0$ implies that $f>0$ in $\Omega_{t}$. We want to find out whether the following problem has a unique solution:

$\square^{2} \phi=\psi(\boldsymbol{x}, t),(\boldsymbol{x}, t) \in \Omega_{t} \times[0, T]$

$\mathrm{IC}: \phi(\boldsymbol{x}, 0)=g(\boldsymbol{x}), \partial_{t} \phi(\boldsymbol{x}, t)=h(\boldsymbol{x}), \boldsymbol{x} \in \Omega_{t}$

$\mathrm{BC}$ : either $\phi(\boldsymbol{x}, t)=k(\boldsymbol{x}, t)$ or $\frac{\partial \phi}{\partial n}=k(\boldsymbol{x}, t)$ is specified for $(\boldsymbol{x}, t) \epsilon \partial \Omega_{t} \times[0, T]$

In this section, we are taking $c=1$ in the wave operator, i.e., $\square^{2}=\partial_{t}^{2}-\nabla^{2}$.

As is well-known from the theory of partial differential equations, the above BCs are called the Dirichlet and Neumann boundary conditions, respectively. We require that the IC and BC be consistent, e.g., for Dirichlet BC, one should have $k(\boldsymbol{x}, 0)=g(\boldsymbol{x})$ on $\partial \Omega_{t}$.

To prove uniqueness, we must show that if $\phi_{1}(\boldsymbol{x}, t)$ and $\phi_{2}(\boldsymbol{x}, t)$ are two solutions of eq.(9), then their difference $\phi=\phi_{1}-\phi_{2}$ which satisfies

$\square^{2} \phi=0, \quad(x, t) \epsilon \Omega_{t} \times[0, T]$

IC $: \phi(x, 0)=0, \partial_{t} \phi(x, 0)=0, x \in \Omega_{t}$

$\mathrm{BC}$ : either $\phi(x, t)=0$ or $\frac{\partial \phi}{\partial n}=0$ for $(\boldsymbol{x}, t) \epsilon \partial \Omega_{t} \times[0, T]$

has the solution $\phi(x, t) \equiv 0$ for $(\boldsymbol{x}, t) \epsilon \Omega_{t} \times[0, T]$.

The general method of proof is based on an energy relation. This is described in Subsection 3.2 below.

\subsection{The Classical Uniqueness Theorem}

The classical uniqueness theorem states that for a stationary surface $f(x)=0$, the solution of eq. (1) is unique ${ }^{45}$. We will not give the proof here because it is a special case of our more general result below.

\subsection{Extension of the Uniqueness Theorem to a Moving Deformable Surface}

We want to show that the solution of problem described by eq.(10) is $\phi(x, t) \equiv 0$ for $(x, t) \epsilon \Omega_{t} \times[0, T]$. We start with the following easily established identity: 
$\phi_{t} \square^{2} \phi=\frac{\partial}{\partial t}\left(\frac{1}{2} \phi_{t}^{2}\right)-\nabla \cdot\left(\phi_{t} \nabla \phi\right)+\nabla \phi_{t} \cdot \nabla \phi=\frac{\partial}{\partial t}\left(\frac{1}{2} \phi_{t}^{2}+\frac{1}{2}|\nabla \phi|^{2}\right)-\nabla \cdot\left(\phi_{t} \nabla \phi\right)$

We now integrate this result for $(x, t) \in \Omega_{t} \times[0, T]$ and use the divergence theorem. This gives

$\int_{0}^{T} \int_{\Omega_{t}} \frac{\partial E}{\partial t} d \boldsymbol{x} d t=\int_{0}^{t} \int_{\partial \Omega_{t}} \phi_{t} \frac{\partial \phi}{\partial n} d S d t$

where $E=\frac{1}{2} \phi_{t}^{2}+\frac{1}{2}|\nabla \phi|^{2}$ which can be interpreted as an energy quantity. We next obtain the following result to use on the left side of the above equation:

$\frac{d}{d t} \int_{\Omega_{t}} E(\boldsymbol{x}, t) d \boldsymbol{x}=\frac{d}{d t} \int_{\mathbb{R}^{3}} H(f) E(\boldsymbol{x}, t) d \boldsymbol{x}=\int_{\mathbb{R}^{3}} H(f) \frac{\partial E(\boldsymbol{x}, t)}{\partial t} d \boldsymbol{x}-\int_{\mathbb{R}^{3}} \mathrm{v}_{n} \delta(f) E d \boldsymbol{x}$

Here $H(f)$ and $\delta(f)$ are the Heaviside function and the Dirac delta functions, respectively. We have also used the relation $\partial f / \partial n=-\mathrm{v}_{n}$ where $\mathrm{v}_{n}$ is the local normal velocity on the surface $f=0$. We next use the following relation proved in references 37,38 in the above equation:

$\int_{\mathbb{R}^{3}} \mathrm{v}_{n} \delta(f) E d \boldsymbol{x}=\int_{\partial \Omega_{t}} \mathrm{v}_{n} E d S$

Equation (13), with some rearrangement, will then yield:

$\int_{\mathbb{R}^{3}} H(f) \frac{\partial E(\boldsymbol{x}, t)}{\partial t} d \boldsymbol{x}=\int_{\Omega_{t}} \frac{\partial E}{\partial t} d \boldsymbol{x}=\frac{d}{d t} \int_{\Omega_{t}} E d \boldsymbol{x}+\int_{\partial \Omega_{t}} \mathrm{v}_{n} E d S$

This is, of course, a transport theorem. We next use this result in eq.(12) and keep the surface integrals on the right of equality sign to get:

$\int_{0}^{T} \frac{d}{d t} \int_{\Omega_{t}} E d \boldsymbol{x} d t=\int_{0}^{T} \int_{\partial \Omega_{t}}\left(\phi_{t} \frac{\partial \phi}{\partial n}-\mathrm{v}_{n} E\right) d S d t$

We next integrate the time integral on the left side. This gives

$\int_{\Omega_{t}(T)} E d \boldsymbol{x}-\int_{\Omega_{t}(0)} E d \boldsymbol{x}=\int_{0}^{T} \int_{\partial \Omega_{t}}\left(\phi_{t} \frac{\partial \phi}{\partial n}-\mathrm{v}_{n} E\right) d S d t$

Here $\Omega_{t}(T)$, and $\Omega_{t}(0)$ are the regions exterior to the surfaces $f(\boldsymbol{x}, T)=0$ and $f(\boldsymbol{x}, 0)=0$, respectively. Now we utilize the IC of eq.(10) in the second integral on the left side of eq.(17) to get

$\int_{\Omega_{t}(0)} E d \boldsymbol{x}=0$

Equation.(17), therefore, becomes

$$
\int_{\Omega_{t}(T)} E d \boldsymbol{x}=\int_{0}^{T} \int_{\partial \Omega_{t}}\left(\phi_{t} \frac{\partial \phi}{\partial n}-\mathrm{v}_{n} E\right) d S d t \quad \text { The Energy Identity }
$$

This is the generalization of the energy identity used for obtaining the classical uniqueness theorem 45 . We now prove some very useful uniqueness theorems based on this identity for moving and deformable surfaces for our applications.

\subsubsection{The Uniqueness Theorem for an Infinitely Thin Deformable Surface in Motion}

For such a body $\mathrm{v}_{n}=0$ so that eq.(19) becomes identical to what that is obtained for stationary surfaces 45 :

$$
\int_{\Omega_{t}(T)} E d \boldsymbol{x}=\int_{0}^{T} \int_{\partial \Omega_{t}} \phi_{t} \frac{\partial \phi}{\partial n} d S d t
$$


Now using either the Dirichlet or the Neumann BC of eq.(10), the right side of eq.(20) vanishes and we obtain:

$$
\int_{\Omega_{t}(T)} E d \boldsymbol{x}=0
$$

Since $E$ is a positive quantity, this relation implies that $E=0$. From this we conclude that $\phi=\operatorname{constant}=\phi(x, 0)=0$.

\subsubsection{The Uniqueness Theorem for an Arbitrary Deformable Surface in Motion With Dirichlet Boundary Condition}

This theorem is considerably more difficult to prove than for an infinitely thin moving body. We will again utilize eq.(19) but this time some concepts from differential geometry must be used to prove uniqueness of the solution. We mention here that by using eq.(19), it is a trivial matter to show that we have uniqueness if consistent Dirichlet and Neumann BCs are specified on a moving surface. In fact, in this case we have a closed form solution which is the Kirchhoff formula for moving surfaces

first derived by Morgans 46 in 1930 and verified much later using generalized function theory by Farassat and Myers 47,48 . We know that in the classical Kirchhoff formula, the imposition of the Dirichlet and Neumann BCs is an overspecification of data (from classical uniqueness theorem given above). We also feel intuitively, based on the fact that there is total destructive interference of acoustic signal inside the moving surface, that giving the Dirichlet and Neumann BCs on a moving surface is also an overspecification. Now that many researchers are advocating the use of EGF for TE noise prediction, there is a need for uniqueness theorems of wave equation for either Dirichlet, Neumann on a moving and deformable surface. We now proceed with the proof of the uniqueness theorem when only the Dirichlet BC is specified on the moving surface.

First we find the expression for the integrand of the surface integral on the right of eq.(19) in terms of the quantities known on the surface. We then proceed to show that the integral on the right of this equation vanishes in the vicinity of any time $t=t_{0}$. This process then can be repeated for all $t \in[0, T]$. This leads to the conclusion that integrand of the integral on the left of eq.(19) is zero. The conclusion of $\phi \equiv 0$ follows by the same reasoning as used in the case of infinitely thin surface.

Note that eq.(19) was derived in a frame fixed to the undisturbed medium. This means that in this equation $\phi_{t}=\partial \phi(\boldsymbol{x}, t) / \partial t$. This is not the same time derivative of $\phi$ on the moving surface. Referring to fig. 4 , we define a Gaussian coordinate system $\left(u^{1}, u^{2}\right)$ on the surface $f=0$. Next define the coordinate $u^{3}$ locally normal to this surface with positive direction along the direction of $\boldsymbol{n}$ with $u^{3}$ as the distance from the surface (time $t$ is frozen). Since $\boldsymbol{n}=\nabla f$, this means simply that we have defined $u^{3}=f$,i.e., $d n=d f=d u^{3}$. We will use the notation $\boldsymbol{u}=\left(u^{1}, u^{2}, u^{3}\right)$. We can then show that, if we define $\tilde{\phi}(\boldsymbol{u}, t)=\phi(\boldsymbol{x}(\boldsymbol{u}, \boldsymbol{t}), t)$

$\tilde{\phi}_{t}(\boldsymbol{u}, t)=\frac{\partial \tilde{\phi}(\boldsymbol{u}, t)}{\partial t}=\frac{\partial \phi(\boldsymbol{x}(\boldsymbol{u}, \boldsymbol{t}), t)}{\partial t}=\phi_{t}(\boldsymbol{x}, t)+\boldsymbol{v} \cdot \nabla \phi(\boldsymbol{x}, t)=\phi_{t}(\boldsymbol{x}, t)+\boldsymbol{v} \cdot \nabla \tilde{\phi}(\boldsymbol{u}, t)$

where $\boldsymbol{v}=\partial \boldsymbol{x}\left(u^{1}, u^{2}, 0, t\right) / \partial t$ is the local surface velocity which is unambiguously defined. Note that the Dirichlet BC can now be written simply as $\tilde{\phi}\left(u^{1}, u^{2}, 0, t\right)=0$. This means that $\nabla \tilde{\phi}\left(u^{1}, u^{2}, 0, t\right)=\tilde{\phi}_{n}\left(u^{1}, u^{2}, 0, t\right) \boldsymbol{n}=\phi_{n}(\boldsymbol{x}, t) \boldsymbol{n}$ where $\tilde{\phi}_{n}=\partial \tilde{\phi} / \partial u^{3}$. Equation (22) and the BC then give

$\left.\phi_{t}(\boldsymbol{x}, t)\right|_{f=0}=-v_{n} \tilde{\phi}_{n}\left(u^{1}, u^{2}, 0, t\right)=-\left.v_{n} \phi_{n}(\boldsymbol{x}, t)\right|_{f=0}$

Using all these results in the integrand of eq.(19), we get

$\left(\phi_{t} \frac{\partial \phi}{\partial n}-\mathrm{v}_{n} E\right)_{f=0}=-v_{n}\left(3+v_{n}^{2}\right) \tilde{\phi}_{n}^{2}\left(u^{1}, u^{2}, 0, t\right)$

The most important thing to recognize at this point is that the expression on the right of eq.(24) is defined in the vicinity of the surface $f=0$ at any time as a function of coordinates $(\boldsymbol{u}, t)$ and not just $\left(u^{1}, u^{2}, 0, t\right)$. We use this fact in eq.(25) below. Now we will take two steps that are crucial in the proof:

1- At the time $t_{0} \in[0, T]$, set up the coordinate system $\boldsymbol{u}$ on the surface $f\left(\boldsymbol{x}, t_{0}\right)=0$ as described above with $\left(u^{1}, u^{2}\right)$ the Gaussian coordinates on this surface and $u^{3}$ coordinate normal to the surface, $u^{3}=0$ on the surface and positive in the direction of $\boldsymbol{n}$ and negative in the opposite direction. Now at the time $t_{0}+\delta \mathrm{t}$, the location of the surface $f\left(\boldsymbol{x}, t_{0}+\delta \mathrm{t}\right)=0$ is obtained by moving each point $\left(u^{1}, u^{2}, 0\right)$ on $f\left(\boldsymbol{x}, t_{0}\right)=0$ to $\left(u^{1}, u^{2}, v_{n} \delta t\right)$. Transfer coordinates $\left(u^{1}, u^{2}\right)$ from $f\left(\boldsymbol{x}, t_{0}\right)=0$ to $f\left(\boldsymbol{x}, t_{0}+\delta \mathrm{t}\right)=0$ by this process and retain the definition of coordinate system $u^{3}$ as defined for surface $f\left(\boldsymbol{x}, t_{0}\right)=0$. 
2- Assume that the limits of the Gaussian coordinates $\left(u^{1}, u^{2}\right)$ in defining the moving surface $f=0$ is independent of time for $t \epsilon\left[t_{0}, t_{0}+\delta \mathrm{t}\right]$. We call this two-dimensional domain $\Omega_{u}$. See Farassat and Myers ${ }^{48}$ for an example. This assumption allows us to exchange the order of integration after the second equality sign in eq.(25) blow.

We can now do all the following manipulations rigorously:

$$
\begin{aligned}
& \int_{t_{0}}^{t_{0}+\delta \mathrm{t}} \quad \int_{\partial \Omega_{t}}\left(\phi_{t} \frac{\partial \phi}{\partial n}-\mathrm{v}_{n} E\right) d S d t=-\int_{t_{0}}^{t_{0}+\delta \mathrm{t}} \int_{\partial \Omega_{u}} v_{n}\left(3+v_{n}^{2}\right) \tilde{\phi}_{n}^{2}\left(u^{1}, u^{2}, u^{3}, t\right) \sqrt{g_{(2)}\left(u^{1}, u^{2}, u^{3}, t\right)} d u^{l} d u^{2} d t= \\
& -\int_{\partial \Omega_{u}} \int_{t_{0}}^{t_{0}+\delta \mathrm{t}} v_{n}\left(3+v_{n}^{2}\right) \tilde{\phi}_{n}^{2} \sqrt{g_{(2)}} d t d u^{l} d u^{2}= \\
& \quad-\int_{\partial \Omega_{u}} \int_{0}^{d n} v_{n}\left(3+v_{n}^{2}\right) \tilde{\phi}_{n}^{2} \sqrt{g_{(2)}} \frac{d n}{v_{n}} d u^{l} d u^{2}=-\int_{\partial \Omega_{u}} \int_{0}^{d n}\left(3+v_{n}^{2}\right)\left(\frac{\delta \tilde{\phi}}{d n}\right)^{2} \sqrt{g_{(2)}} d n d u^{l} d u^{2}=0
\end{aligned}
$$

Here $g_{(2)}$ is the determinant of the coefficients of the first fundamental form ${ }^{49}$. In the last step, we have used $\delta \tilde{\phi}=\tilde{\phi}\left(u^{1}, u^{2}, v_{n} \delta t, t_{0}+\delta \mathrm{t}\right)-\tilde{\phi}\left(u^{l}, u^{2}, 0, t_{0}\right)=0-0=0$ by the BC. We can now repeat the process again for all time $t \epsilon[0, T]$ to obtain:

$$
\int_{\Omega_{t}(T)} E d x=0 \Rightarrow E=\frac{1}{2} \phi_{t}^{2}+\frac{1}{2}|\nabla \phi|^{2}=0 \Rightarrow \phi(x, t)=\text { constant }=\phi(x, 0)=0
$$

Thus the uniqueness of the solution of the wave equation with Dirichlet BC specified on a deformable body in motion is proved.

\subsubsection{The Uniqueness Theorem for an Arbitrary Deformable Surface in Motion With Neumann Boundary Condition}

The basic idea of proof remains the same as in the case of the Dirichlet $\mathrm{BC}$ with some minor differences. Note that we have the Neumann $\mathrm{BC} \phi_{n}=0$ on $\partial \Omega_{t}$. We can show that, after using the $\mathrm{BC}$, we have

$$
\left(\phi_{t} \frac{\partial \phi}{\partial n}-\mathrm{v}_{n} E\right)_{f=0}=-v_{n}\left[\tilde{\phi}_{t}^{2}-2 v_{T} \phi_{T} \tilde{\phi}_{t}+\left(1+v_{T}^{2}\right) \phi_{T}^{2}\right]
$$

where $\boldsymbol{v} \cdot \nabla_{T} \phi=v_{T} \phi_{T}$ where $v_{T}$ and $\phi_{T}$ are the tangential velocity of the surface and the directional derivative of $\phi$ in the direction of the tangential velocity, respectively. We now repeat the procedure for Dirichlet BC except that we should start from $t=0$ and use the fact that $\phi_{n}=0$ to show that $\phi=0$ is transferred at each step from $f(\boldsymbol{x}, t)=0$ to the surface $f(\boldsymbol{x}, t+\delta \mathrm{t})=0, t \in[0, T]$, so that the right side of eq.(27) always vanishes. Equation (26) and the uniqueness result for the Neumann BC then follow.

Remarks-1- Since our reasoning is local, the above method can also establish the uniqueness of the solution of the wave equation with the Robin BC (a linear combination of the unknown and its normal derivative).

2- To the best of our knowledge, the above theorems are proved for the first time here. We have extensively searched mathematics and physics literature and have not found the proofs of these theorems elsewhere. Note that the complications and difficulties in the proof come from the motion of the surface.

3- The above uniqueness theorems ensure the existence of EGF for the problems under consideration. The reason is as follows. For a linear operator, the existence of Green's function is guaranteed if zero is not an eigenvalue of the operator. When zero is an eigenvalue of the operator, it means that uniqueness breaks down. Since we have established uniqueness, zero cannot be an eigenvalue of the problem and the EGF exists. 


\subsection{The solution of the Lighthill Equation with the Dirichlet or the Neumann BC using EGF}

For completeness and for the purpose of comparison of noise prediction formulas in AFN prediction methodologies, we give the solution of Lighthill's equation using EGF. We assume that the moving surface $f=0$ is deformable. The two initialboundary value problems that we will consider are:

$\square^{2} p^{\prime}=\frac{\partial^{2} T_{i j}}{\partial x_{i} \partial x_{j}} \quad x \in \Omega_{t}, t \in[0, T]$,

IC : $p^{\prime}(\boldsymbol{x}, 0)=g(\boldsymbol{x}), \frac{\partial p^{\prime}(\boldsymbol{x}, 0)}{\partial t}=h(\boldsymbol{x}) \quad \boldsymbol{x} \in \Omega_{t}$,

$\mathrm{BC}: p^{\prime}(\boldsymbol{x}, t)=k(\boldsymbol{x}, t) \quad \boldsymbol{x} \in \partial \Omega_{t}, t \in[0, T] \quad$ Dirichlet BC

and

$\square^{2} p^{\prime}=\frac{\partial^{2} T_{i j}}{\partial x_{i} \partial x_{j}} \quad x \in \Omega_{t}, t \in[0, T]$,

IC : $\quad p^{\prime}(\boldsymbol{x}, 0)=g(\boldsymbol{x}), \frac{\partial p^{\prime}(\boldsymbol{x}, 0)}{\partial t}=h(\boldsymbol{x}) \quad x \in \Omega_{t}$,

$\mathrm{BC}: \frac{\partial p^{\prime}(\boldsymbol{x}, t)}{\partial n}=k(\boldsymbol{x}, t) \quad \boldsymbol{x} \in \partial \Omega_{t}, t \in[0, T] \quad$ Neumann BC

We are considering initial-boundary value problems here. The EGFs for the above wave equations satisfy the following conditions:

$\square_{(\boldsymbol{x}, t)}^{2} G(\boldsymbol{x}, \boldsymbol{y}, t-\tau)=\delta(\boldsymbol{x}-\boldsymbol{y}) \delta(t-\tau) \quad \boldsymbol{x} \in \Omega_{t}, \boldsymbol{y} \in \Omega_{t}, \tau<t, \quad t-\tau \in[0, T]$

$G(x, y, t-\tau)=0 \quad x \in \Omega_{t}, y \in \Omega_{t}, \tau>t$

$G(\boldsymbol{x}, \boldsymbol{y}, t-\tau)=0 \quad \boldsymbol{x} \epsilon \partial \Omega_{t}, \boldsymbol{y} \epsilon \Omega_{t}, t-\tau \epsilon[0, T] \quad$ For Dirichlet BC

and

$\square_{(x, t)}^{2} G(\boldsymbol{x}, \boldsymbol{y}, t-\tau)=\delta(\boldsymbol{x}-\boldsymbol{y}) \delta(t-\tau) \quad \boldsymbol{x} \in \Omega_{t}, \boldsymbol{y} \in \Omega_{t}, \tau<t, t-\tau \in[0, T]$

$G(x, y, t-\tau)=0 \quad x \in \Omega_{t}, y \in \Omega_{t}, \tau>t$

$\frac{\partial G(\boldsymbol{x}, \boldsymbol{y}, t-\tau)}{\partial n_{x}}=0 \quad \boldsymbol{x} \in \partial \Omega_{t}, \boldsymbol{y} \in \Omega_{t}, t-\tau \in[0, T] \quad$ For Neumann BC

The solutions of eqs. (28) and (29) are:

$$
\begin{aligned}
& p^{\prime}(\boldsymbol{x}, t)=\int_{0}^{t} \int_{\Omega_{t}(\tau)} \frac{\partial^{2} T_{i j}}{\partial y_{i} \partial y_{j}} G(\boldsymbol{x}, \boldsymbol{y}, t-\tau) d y d \tau- \\
& \int_{\Omega_{t}(0)}\left[g(\boldsymbol{y}) \frac{\partial G(\boldsymbol{x}, \boldsymbol{y}, t-\tau)}{\partial \tau}-h(\boldsymbol{y}) G(\boldsymbol{x}, \boldsymbol{y}, t-\tau)\right] d \boldsymbol{y}+\int_{0}^{t} \int_{\partial \Omega_{t}(\tau)} k(\boldsymbol{y}, \tau) \frac{\partial G(\boldsymbol{x}, \boldsymbol{y}, t-\tau)}{\partial n_{\boldsymbol{y}}} d S_{\boldsymbol{y}} d \tau \text { For Dirichlet BC } \\
& p^{\prime}(\boldsymbol{x}, t)=\int_{0}^{t} \int_{\Omega_{t}(\tau)} \frac{\partial^{2} T_{i j}}{\partial y_{i} \partial y_{j}} G(\boldsymbol{x}, \boldsymbol{y}, t-\tau) d y d \tau- \\
& \quad \int_{\Omega_{t}(0)}\left[g(\boldsymbol{y}) \frac{\partial G(\boldsymbol{x}, \boldsymbol{y}, t-\tau)}{\partial \tau}-h(\boldsymbol{y}) G(\boldsymbol{x}, \boldsymbol{y}, t-\tau)\right] d \boldsymbol{y}+\int_{0}^{t} \int_{\partial \Omega_{t}(\tau)} k(\boldsymbol{y}, \tau) G(\boldsymbol{x}, \boldsymbol{y}, t-\tau) d \boldsymbol{S}_{\boldsymbol{y}} d \tau \quad \text { For Neumann BC}
\end{aligned}
$$

In these equations the region $\Omega_{t}(\tau)$ is external to the surface $\partial \Omega_{t}(\tau): f(\boldsymbol{y}, \tau)=0$. We have left out all the details of the derivation which involve some subtleties. For example, one has to use the transport identity of eq. (15) in the derivation of these solutions. In addition, some of the properties of the Green's function on either side of $\tau=t$ must be exploited (See the books by Goldstein ${ }^{11}$, Stakgold ${ }^{46}$ and Morse and Feshbach ${ }^{52}$ ). 
We mention here that in acoustic applications, it is usually assumed that the noise generation process startes at $t=-\infty$, or at a finite time, after any transient radiation has subsided. Therefore, one rarely sees the volume integrals on the right of eqs.(32) and (33), which account for initial conditions (IC), in current noise prediction formulas. This assumption is made here as well.

\section{Implications of the Uniqueness Theorem}

From eq.(33), we may conclude that, in the case of a stationary airfoil, when the Neumann BC $\partial p^{\prime} / \partial n=0$ is appropriate, the acoustic pressure for a TE noise prediction is given by

$$
p^{\prime}(\boldsymbol{x}, t)=\int_{-\infty}^{t} \int_{\Omega_{t}(\tau)} \frac{\partial^{2} T_{i j}}{\partial y_{i} \partial y_{j}} G(\boldsymbol{x}, \boldsymbol{y}, t-\tau) d y d \tau
$$

This equation is identical Lighthill's equation for jet noise prediction, except that Green's function in the above equation is the EGF that satisfies eq.(31). Therefore, as suggested by Howe, we are justified in using incompressible turbulent flow simulation near the TE to model the source in the integral above. In addition, statistical analysis on this equation to relate the statistics of the noise to the statistics of the turbulence is relatively easy ${ }^{3}$. A word of caution is in order here, as the simplicity of this equation should be balanced against the difficulty of obtaining the EGF, both analytically and numerically.

Experimental and computational evidence indicate that even at high frequencies the wavefronts of the acoustic waves spreading from the TE area are normal to the airfoil surface (see fig. 5) in many TE noise problems. This means that the assumption of the vanishing of the normal derivative of the surface pressure is a reasonable one and use eq.(34) is appropriate for noise prediction at high frequencies and when the airfoil is in motion. Therefore, the use of EGF with the Neumann BC $\partial p^{\prime} / \partial n=0$ offer clear advantages over the use of FSGF in FW-H equation.

Clearly, eq.(34) can be used to calculate broadband noise from turbulent flows over a thin surface moving in its plane. This application has not been explored although there may be situations where such a model can be utilized.

In the general cases where we cannot use $\partial p^{\prime} / \partial n=0$, we must specify $\partial p^{\prime} / \partial n$ on the surface for the Neumann BC. The condition on the EGF is then $\partial G / \partial n=0$ on the boundary of the moving surface. One then does not require the surface pressure data as input. But the noise radiation formula, eq.(33) will have both volume and surface integrals just as the solution of FW-H equation! There does not appear any advantage of EGF over FSGF.

When we use the Dirichlet BC, the surface pressure should be specified on the moving surface and the EGF satisfies $G=0$ on this surface. The radiation formula is eq.(32) which has both surface and volume integrals. In this case, the input data for both the solution of FW-H equation and eq.(32) are identical. There is the possibility that when EGF is utilized, the cancellation between the volume and surface integrals is less than in the case of using FSGF ${ }^{39}$. If such a possibility were proved valid, this would imply less sensitivity in an EGF formulation to the accuracy of the input flow data.

The current experience with the EGF approach has already encountered some limitations in the application of this method in aeroacoustics. Doing analytical work on the EGF, and evaluating it numerically on a computer, incurs many problems, such as singularities on the surface. Furthermore, for complicated surfaces of interest in AFN prediction, the EGF can only be obtained numerically. Therefore, any realistic AFN calculation with an EGF formulation will involve intensive computer usage. It should be mentioned here that these calculations are far less than that for turbulent flow simulations (LES and DNS). Thus, in general, the use of EGF is within our reach today. Therefore, we think that the use of EGF should be explored further for Direichlet and Neumann BCs. 


\section{Exact Green's Functions for a Wing in Uniform Rectilinear Motion}

Much efforts has been spent to find either analytically 3,4 or numerically ${ }^{5-9}$ the EGF for nontrivial geometries. The most significant lesson that one learns from these works is that constructing EGF either analytically or numerically is difficult. With the exception of the article by $\mathrm{Hu}$, Guo and Jones ${ }^{9}$ EGFs have been constructed for two-dimensional geometries. However, Dunn and Tinetti have developed the Fast Scattering Code (FSC) to calculate the scattering of the engine noise from thin bodies in motion 50,51 . The FSC solves the Helmholtz equation for a surface in uniform rectilinear motion on which the condition $\partial p^{\prime} / \partial n=0$ or an absorbing BC is imposed. In a typical FSC simulation, an incident acoustic source is specified off the surface to model the propulsion system noise. The scattered acoustic field is calculated by the efficient equivalent source method. The problem is solved in the frame moving with the surface so that the complex pressure amplitude on and off the moving surface can be found in the moving frame when a source with the complex time dependence of $e^{i \omega t}$ is specified. Therefore, if the incident noise source is taken as a constant frequency monopole source with unit strength fixed in the moving frame, the pressure distribution on the moving surface gives the complex EGF in the moving frame.

We present results for two three dimensional wings with realistic geometries. The designs and dimensions of these wings are shown in figs. 6 and 7. Figures 8 and 9 show the locations of sources for which EGF on the wing surface is computed. The radius of the circle of the sources which is located in a plane normal to the wing is the mean chord length for each wing. Each source is spaced 10 degrees apart on the circle. The zero degree is the location ahead of the LE, 90 degrees is exactly above the suction side and 270 degrees is exactly below the pressure side of the wing. We only present results for three source positions for each wing at two frequencies of $3000 \mathrm{~Hz}$ and $4097 \mathrm{~Hz}$ in the figs. 10 to 13.

The values plotted in these surface distributions are the absolute value of the complex amplitude of EGF on the wings for the observer at the assumed source position. In other words, we are presenting in color $|G(\boldsymbol{x}, \boldsymbol{y}, t-\tau)|=\left|\tilde{G}(\boldsymbol{x}, \boldsymbol{y}) e^{i \omega(t-\tau)}\right|=|\tilde{G}(\boldsymbol{x}, \boldsymbol{y})|$ for the observer $\boldsymbol{x}$ at the source position and the field point $\boldsymbol{y}$ varying over all the points on the wing surface. Therefore, we have high sensitivity of the noise to the sources on the regions of the wing that have high magnitudes (white or red) at the observer position. In particular, we see that for some observer positions, the noise is sensitive to the sources over the trailing edge. Note that only a limited range of TE sources contributes to the noise at the observer positions shown. This graphical representation of EGF on the wing can serve in identifying the directivity of sources on specific areas of the wing by varying $\boldsymbol{x}$, or given the observer position, we can identify regions of the wing that radiate toward the observer at $\boldsymbol{x}$.

The FSC is a highly efficient method of calculating EGF of the wave equation with the Neumann BC. It gives aeroacousticians yet another tool to predict AFN.

\section{Concluding Remarks}

In this paper, preliminary results are presented for a study involving a critical examination of the potential use of EGF formulations for the prediction of broadband noise due to turbulent flow over an airfoil surface and its TE. In the process of studying this problem we have proved that for a moving deformable surface, infinitely thin or with thickness, the initialboundary value problem of the wave equation has a unique solution for either the Dirichlet of the Neumann BC on the surface. This existence of EGF then follows from these theorems.

Our conclusions concerning the use of EGF are:

1- For a stationary surface, the use of EGF for TE noise prediction with the Neumann BC $\partial p^{\prime} / \partial n=0$ results in a volume integral involving the quadrupoles in the vicinity of TE. An incompressible turbulent flow simulation near the TE is sufficient for low frequencies but compressible turbulent flow simulation may be needed for high frequencies.

2- It appears that the same Neumann BC $\partial p^{\prime} / \partial n=0$ can be used for TE noise prediction for an airfoil in motion at higher Mach numbers. The choice of compressibility of turbulent flow simulation near the TE must be decided by the frequency range of TE noise and the speed of the airfoil. 
3- The use of EGF with Neumann BC makes the statistical analysis of TE noise much simpler than when FSGF is used. This conclusion applies only when we can use the $\mathrm{BC} \partial p^{\prime} / \partial n=0$ on the airfoil surface. Otherwise, no advantage over the use of FSGF can be realized.

4- In the situations where the condition $\partial p^{\prime} / \partial n=0$ does not apply, the radiation formulas for the Dirichlet and the Neumann $\mathrm{BC}$ have both volume and surface integrals. No clear advantage of the use of EGF over FSGF can be seen as to the amount of input data and ease of statistical analysis.

5- The use of EGF with the Dirichlet BC has not been fully explored. The input data needed are identical to when FSGF is used to solve FW-H equation. The only possible advantage that one can foresee is the reduction of cancellation between the volume and surface integrals as compared to the use of FW-H equation. Because of the availability of the method based on $\mathrm{FW}-\mathrm{H}_{\mathrm{pds}}$ and the present difficulty of getting EGF, perhaps this advantage will not give enough incentive to researchers to examine such an approach.

6- We have not been able to discern any advantages for EGF for predicting the noise from turbulent BL over an airfoil although for a stationary surface, the ease of statistical analysis of the broadband noise when the Neumann $\mathrm{BC} \partial p^{\prime} / \partial n=0$ is used may be an advantage over the use of FSGF.

7- We feel that the use of EGF in AFN prediction is still an open problem. We, therefore, recommend that the use of EGF be explored further in acoustics.

\section{Acknowledgements}

The first author thanks Professor Michael K. Myers of The George Washington University for many fruitful discussions on the uniqueness theorems for wave equation for moving surfaces. He is also grateful to Professor P. J. Morris of Penn State for some useful discussions concerning the use of EGF for the prediction of broadband noise.

\section{References}

1. F. Farassat and Jay H. Casper: Towards an airframe noise prediction methodology- Survey of current approaches, AIAA2006-0210, Presented at AIAA 44th Aerospace Sciences Meeting and Exhibit, Reno, Nevada, January 9-12, 2006

2 M. J. Lighthill: On sound Generated aerodynamically. I. General Theory, Proceedings of the Royal Society of London, A $211,1952,564-587$.

3. P. J. Morris and F. Farassat: On the acoustic analogy and alternative theories of jet noise prediction, AIAA Journal, 40 (4),2002, 671-680

4. J. E. Ffowcs Williams and D. L. Hawkings: Sound generated by turbulence and surfaces in arbitrary motion, Philosophical Transactions of the Royal Society, A264, 1969, 321-342

5. M. S. Howe: Trailing edge noise at low Mach numbers, Journal of Sound and Vibration, 225, 1999, $211-238$

6. M. S. Howe: Edge-source acoustic Green's function for an airfoil of arbitrary chord, with application to trailing-edge noise, Quarterly Journal of Mechanics and Applied Mathematics, 54, 2001, 139-155

7. M. S. Howe: Theory of Vortex Sound, Cambridge University Press, 2003

8. E. Manoha, G. Elias, B. Troff and P. Sagut: Toward the use of boundary element method in computational aerodynamics, AIAA-99-1980, 5th AIAA/CEAS Aeroacoustics Conference and Exhibit, Bellevue, WA, May 10-12, 1999

9. J. S. D. Ostertag, S. Guidati, G. Guidati, S. Wagner, A. Wilde, and N. Kalitzin: Prediction and measurement of airframe noise on a generic body, AIAA 2000-2063, 6th AIAA/CEAS, Aeroacoustics Conference and Exhibit, Lahaina, HI, 12-14 June 2000

10. X. Gloerfelt, F. Pérot, C. Bailly and D. Juvé: Flow-induced cylinder noise formulated as a diffraction problem for low Mach numbers, Journal of Sound and Vibration, 287, 2005, 129-151 
11. Michel Roger and Stéphane Moreau: Back-scattering correction and further extension of Amiet's trailing-edge noise model. Part 1: theory, Journal of Sound and Vibration, 286, 2005, 477-506

12. F. Q. Hu, Y. P. Guo and A.D. Jones: On the computation and application of exact Green's function in acoustic analogy, 11th AIAA/CEAS Aeroacoustics Conference, Monterey, CA; USA; 23-25 May 2005

13. Marvin Goldstein: Unified approach to aerodynamic sound generation in the presence of solid boundaries, Journal of Acoustical Society of America, 56(2), 1974, 497-509

14. Marvin E. Goldstein: Aeroacoustics, McGraw-Hill Book Company, 1976

15- R. K. Amiet: Acoustic radiation from an airfoil in a turbulent stream, Journal of Sound and Vibration, 41(4), 1975, 407420

16- R. K. Amiet: Noise due to turbulent flow past a trailing edge, Journal of Sound and Vibration,47(3), 1976, 387-393

17- R. K. Amiet: A Note on edge noise theories, Journal of Sound and Vibration, 78(4), 1981, 485-488

18- Robert W. Paterson and Roy K. Amiet: Acoustic radiation and surface pressure characteristics

of an airfoil due to incident turbulence, NASA Contractor Report CR-2733, 1976

19- Robert H. Schlinker and R. K. Amiet: Helicopter rotor trailing edge noise, NASA Contractor report CR-3470, 1981

20- M. S. Howe: A review of theory of trailing edge noise, Journal of Sound and Vibration, 61, 1978, 437-465

21- M. S. Howe: Trailing edge noise at low Mach numbers, Part 2: Attached and separated edge flows, Journal of Sound and Vibration, 234, 2000, 761-775

22- T. F. Brooks and C. L. Burley: Rotor broadband noise prediction with comparison to model data, AIAA Paper 20012210, AIAA/CEAS Aeroacoustics Conference and Exhibit, Maastricht, Netherlands, May 28-30, 2001

23- C. L. Burley and T. F. Brooks, M. A. Marcolini, A. G. Brand and D. A. Conner: Tiltrotor aeroacoustic code (TRAC) predictions and comparisons with measurements, Journal of American Helicopter Society, 45(2), 2000, 80-89

24- T. F. Brooks, and William M. Humphreys, Jr., Flap edge aeroacoustic measurements and predictions, Journal of Sound and Vibration, 261, 2003, 31-74

25- J. E. Ffowcs Williams and L. H. Hall. Aerodynamic sound generation by turbulent

flow in the vicinity of a scattering half plane, Journal of Fluid Mechanics, 40, 1970, 657-670

26- D. G. Crighton, Radiation from vortex filament motion near a half-plane, Journal

of Fluid Mechanics, 51, 1972, 357-362

27- Bart A. Singer, Kenneth S. Brentner, David P. Lockard, and Geoffrey M. Lilley: Simulation of acoustic scattering from a trailing edge, Journal of Sound and Vibration, 230(3), 2000, 541-560

28- Marvin E. Goldstein: Aeroacoustics, NASA SP-346, 1974, available on NASA report server

29- D. G. Crighton, A. P. Dowling, J. E. Ffowcs Williams, M. Heckl and F. G. Leppington: Modern Methods in Analytical Acoustics- Lecture Notes, Springer-Verlag, 1992

30- Kenneth S. Brentner and F. Farassat: An analytical comparison of the acoustic analogy and Kirchhoff formulation for moving surfaces, AIAA Journal, 36(8), 1998, 1379-1386

31- C.L. Streett, D.P. Lockard, B.A. Singer, M.R. Khorrami, and M.M. Choudhari: In search of physics: The interplay of experiment and computation in airframe noise research; flap-edge noise, AIAA 2003-0979, 41st Aerospace Sciences Meeting \& Exhibit, January 6-9, 2002, Reno, Nevada

32- Mehdi R. Khorrami, Meelan Choudhari, Bart A. Singer, David P. Lockard, and Craig L. Streett: In search of the physics: The interplay of experiment and computation in slat aeroacoustics, AIAA 2003-0980, 41st Aerospace Sciences Meeting \& Exhibit, January 6-9, 2002, Reno, Nevada 
33- David P. Lockard and Geoffrey M. Lilley: The airframe noise reduction challenge, NASA TM-2004-213013, 2004

34- Bart A. Singer and Yueping Guo: Development of computational aeroacoustics tools for airframe noise calculations, International Journal of Computational Fluid Dynamics, 18 (6), 2004, 455-469

35- Meng Wang, Jonathan B. Freund and Sanjiva K. Lele: Computational Prediction of Flow-Generated Sound, Annual Reviews of Fluid Mechanics, 38, 2006, 483-512

36- B. A. Singer, D. P. Lockard and G. M. Lilley: Hybrid acoustic prediction, International Journal of Computers \& Mathematics with Applications, 46, 2003, 647-669

37- F. Farassat, Introduction to Generalized Functions With Applications in Aerodynamics and Aeroacoustics, May 1994 (Corrected April 1996), NASA Technical Paper 3428

38- F. Farassat: The Kirchhoff Formulas for Moving Surfaces in Aeroacoustics - The Subsonic and Supersonic Cases, NASA Technical Memorandum 110285, September 1996

39- Jay H. Casper, David P. Lockard, Mehdi Khorrami and Craig Streett: Investigation of volumetric sources in airframe noise simulations, AIAA-2004-2805, 10th AIAA/CEAS Aeroacoustics Conference , Manchester, United Kingdom, May $10-12,2004$

40- F. Farassat and K. S. Brentner: The Uses and Abuses of the Acoustic Analogy in Helicopter Rotor Noise Prediction. Journal of the American Helicopter Society, 33, 1988, 29-36

41- F. Farassat and M. K. Myers: An Analysis of the Quadrupole Noise Source of High Speed Rotating Blades, Computational Acoustics - Scattering, Gaussian Beams, and Aeroacoustics, D. Lee, A. Cakmak, R Vichnevetsky, Editors, NorthHolland Publishing Company, Amsterdam, Vol. 2, 1990, 227-240

42- N. Curle: The influence of solid boundaries upon aerodynamic sound, Proceedings of the Royal Society of London, A231, 1955, 505-514

43- A. Powell: Aerodynamic noise and the plane boundary, Journal of the Acoustical Society of America, 32, 1960, $962-990$

44- Allan D. Pierce: Acoustics-An Introduction to Its Physical Principles and Applications, Acoustical Society of America, 1989

45- Ivar Stakgold: Boundary Value Problems of Mathematical Physics, Volume 2, Macmillan, 1968

46- W. R. Morgans: The Kirchhoff formula extended to a moving surface, Philosophical Magazine, 9, 1930, $141-161$.

47- F. Farassat and M. K. Myers: Extension of Kirchhoff's formula to radiation from moving sources, Journal of Sound and Vibration, 123 (3), 1988, 451-460

48- F. Farassat: The Kirchhoff Formulas for Moving Surfaces in Aeroacoustics - The Subsonic and Supersonic Cases, NASA Technical Memorandum 110285, September 1996

49- Rutherford Aris: Vectors, Tensors and the Basic Equations of Fluid Mechanics, Dover Books on Engineering, 1990

50- Mark H. Dunn and Ana F. Tinetti: Aeroacoustic Scattering Via The Equivalent Source Method, AIAA-2004-2937, 10th AIAA/CEAS Aeroacoustics Conference, Manchester, United Kingdom, May 10-12, 2004

51- Ana F. Tinetti and Mark H. Dunn: Aeroacoustic Noise Prediction Using the Fast Scattering Code, AIAA 2005-3061, 11th Aeroacoustics Conference, Monterey, California, May 23-25, 2005

52- Philip M. Morse and Herman Feshbach: Methods of Theoretical Physics, Volume 1, McGraw-Hill Book Company, 1953 


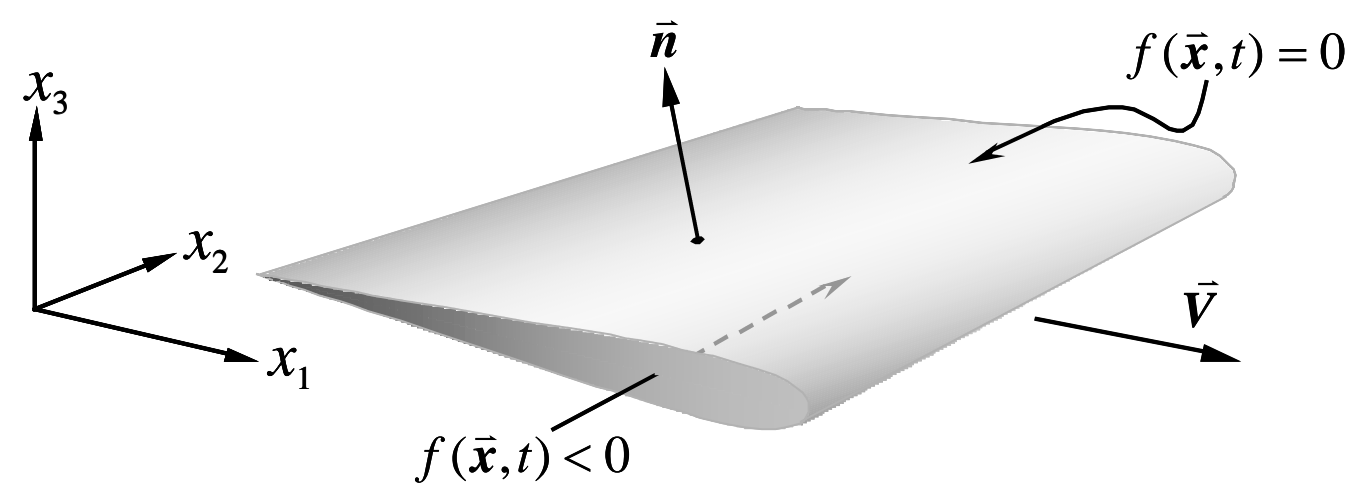

Figure 1. Description of a surface in motion by $f(\overrightarrow{\boldsymbol{x}}, t)=0, \overrightarrow{\boldsymbol{n}}=\vec{\nabla} f$, and $f(\overrightarrow{\boldsymbol{x}}, t)>0$ outside the surface.

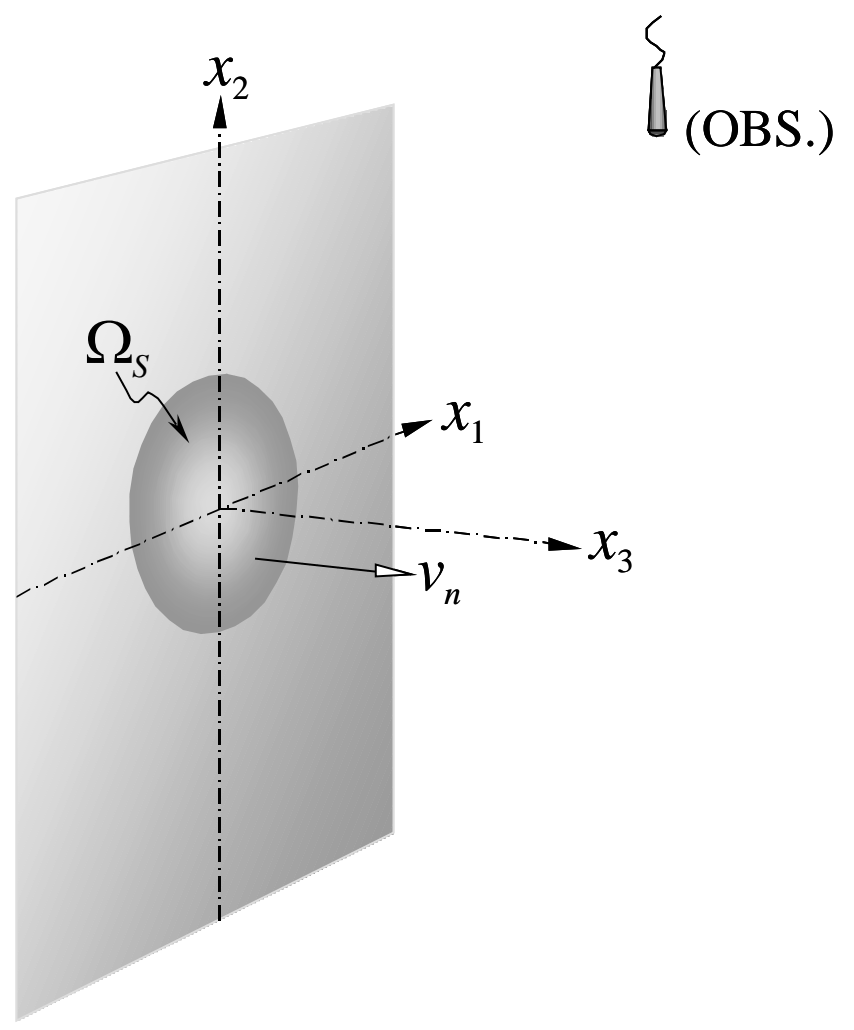

Figure 2. Piston in the wall problem. The darker shaded region is vibrating 


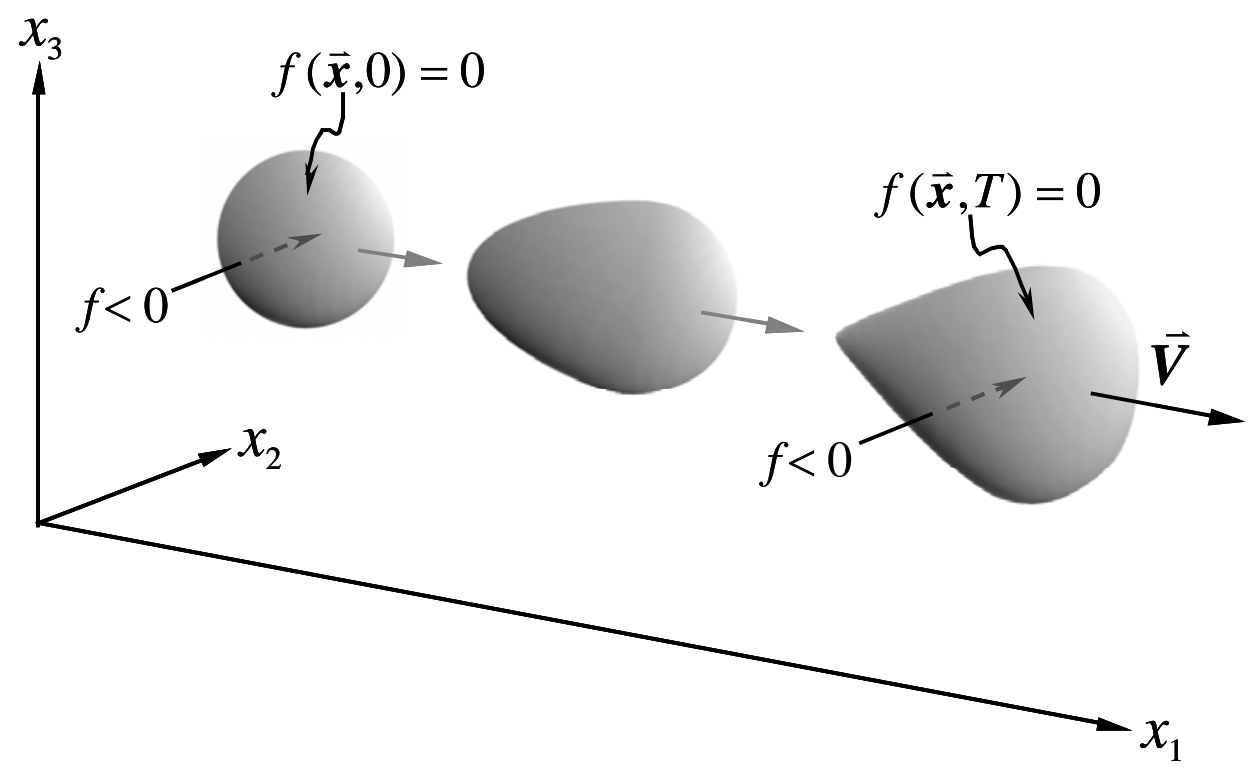

Figure 3. Deformable surface in motion described by $f(\overrightarrow{\boldsymbol{x}}, t)=0, \overrightarrow{\boldsymbol{n}}=\vec{\nabla} f$, and $f(\overrightarrow{\boldsymbol{x}}, t)>0$ outside the surface.

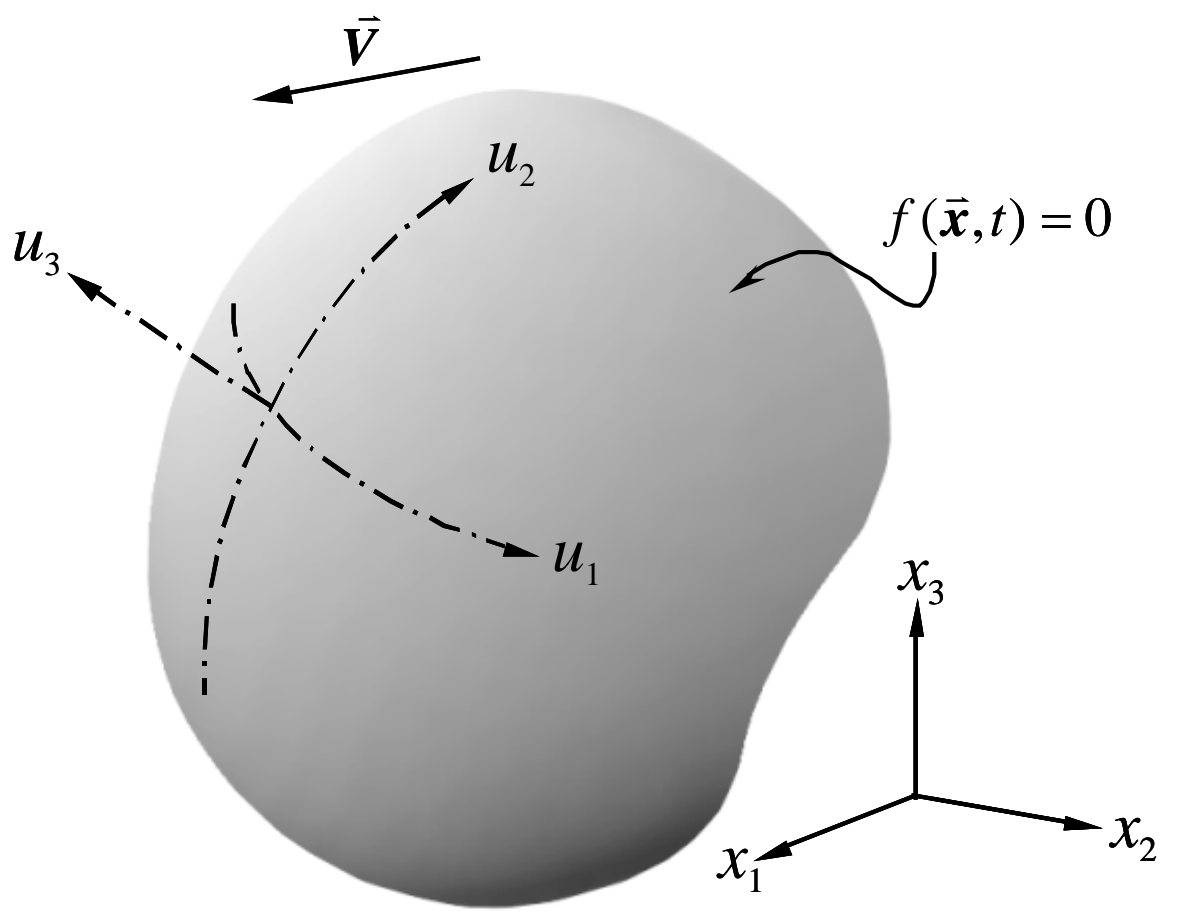

Figure 4. Gaussian coordinate system on a deformable body in motion. 


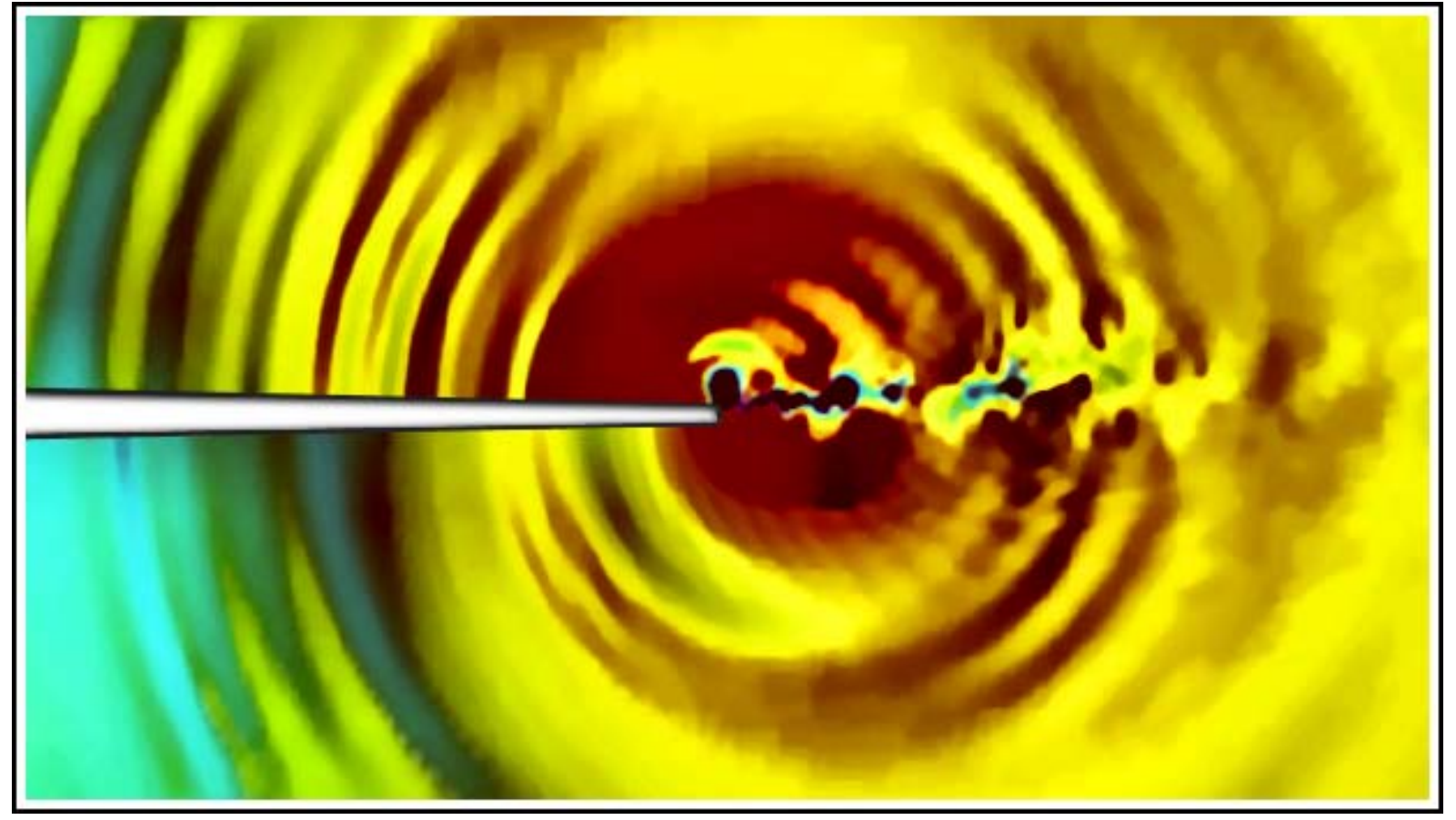

Figure 5. Radiation of waves from a TE, showing the wavefronts propagating normal to the airfoil surface. 


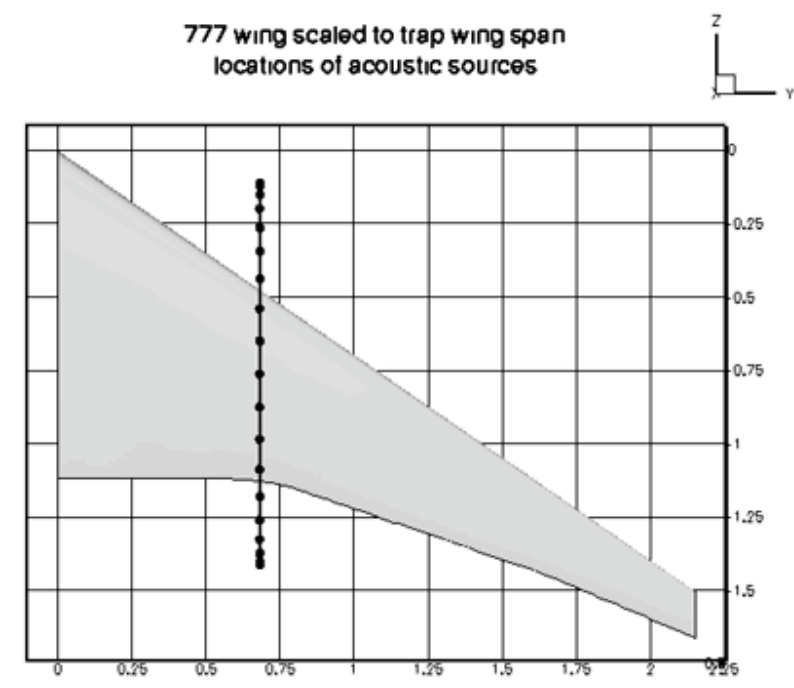

Figure 6. The planform of model 777 wing used for Green's function calculations. The plane of the source (observer) positions is also shown.

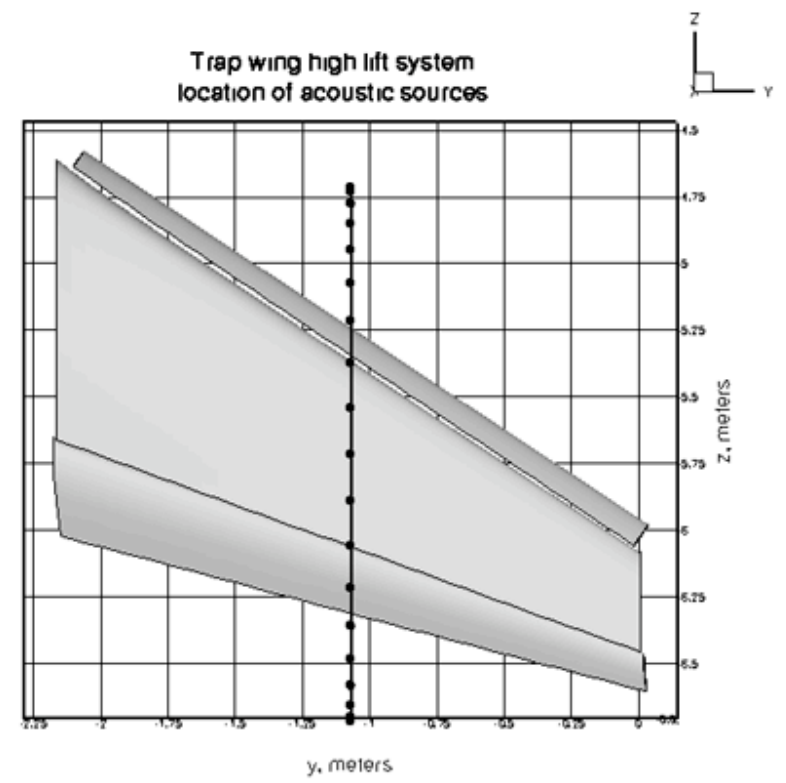

Figure 7. The planform of model Trap Wing used for Green's function calculations. The plane of the source (observer) positions is also shown. 


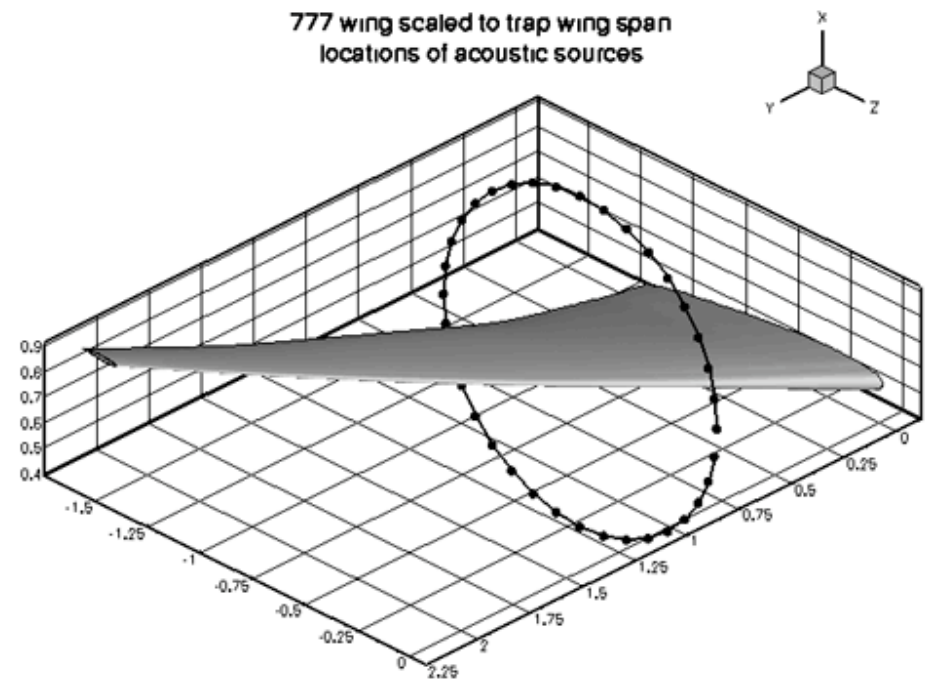

Figure 8. The source (Observer) positions around a circle in a plane for 777 wing. Only the EGF for three observer positions are reported here.

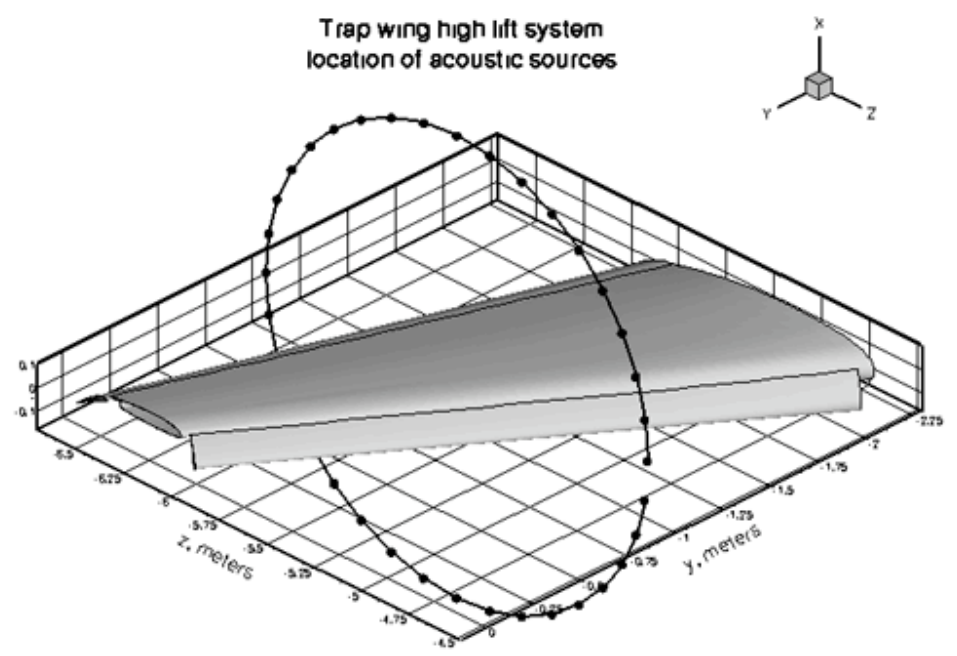

Figure 9. The source (Observer) positions around a circle in a plane for the Trap Wing. Only the EGF for three observer positions are reported here. 


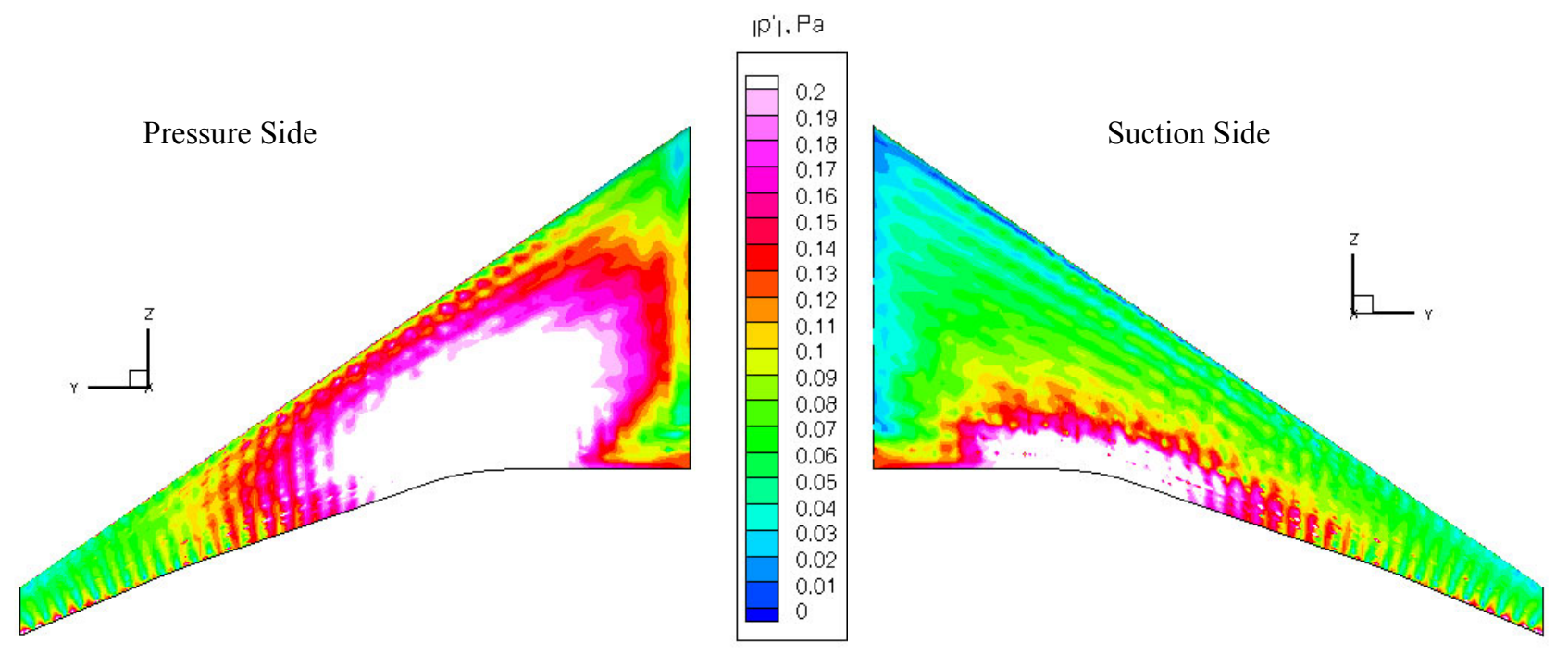

(a) Monopole acoustic source at $190^{\circ}$ from horizontal.
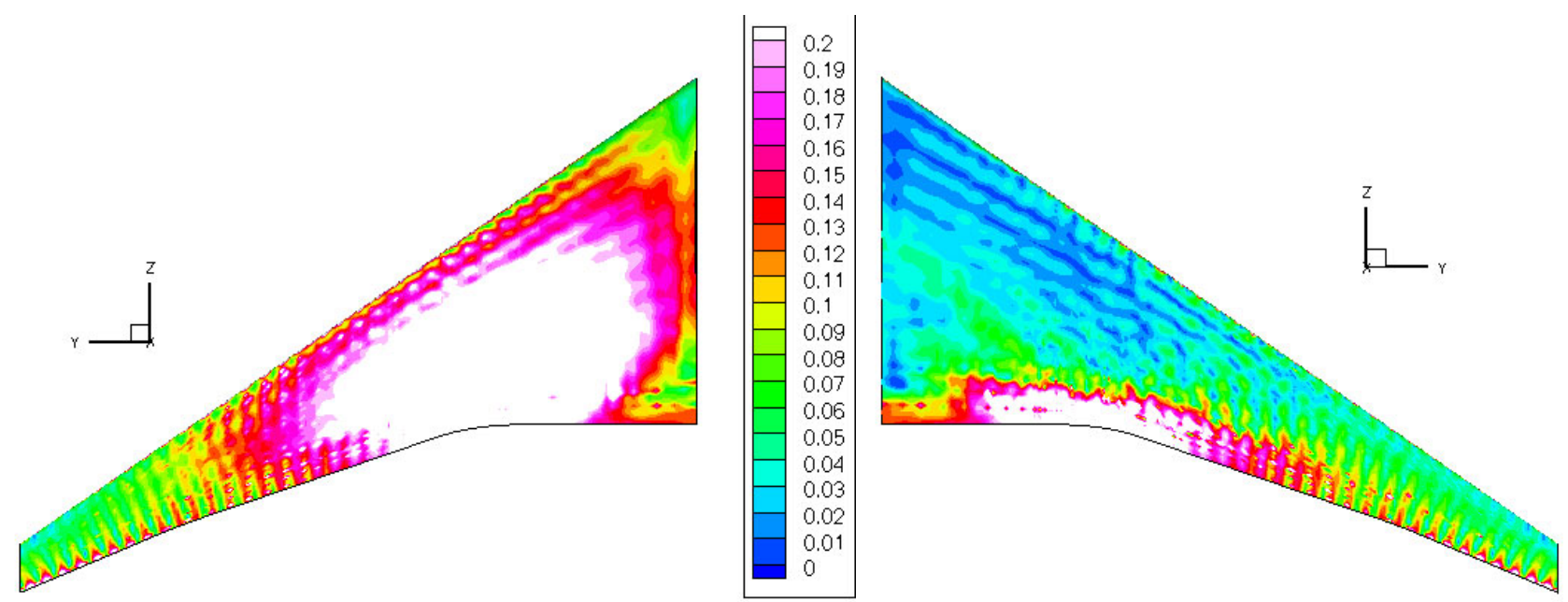

(b) Monopole acoustic source at $200^{\circ}$ from horizontal.
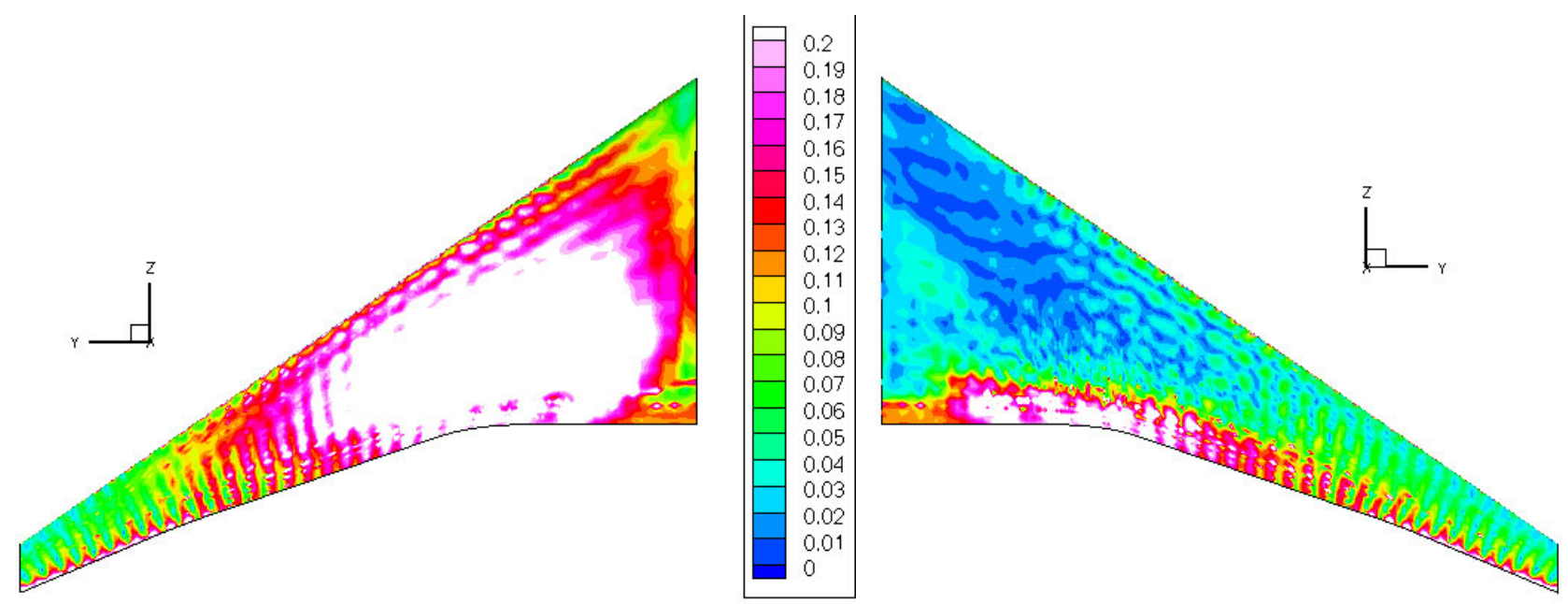

(c) Monopole acoustic source at $210^{\circ}$ from horizontal.

Figure 10 - Magnitude of acoustic pressure $(\mathrm{Pa})$ on scaled Boeing 777 wing $(\mathrm{b} / 2=2.15 \mathrm{~m})$, $\mathrm{f}=3000 \mathrm{~Hz}, \mathrm{M}=0.2$. 


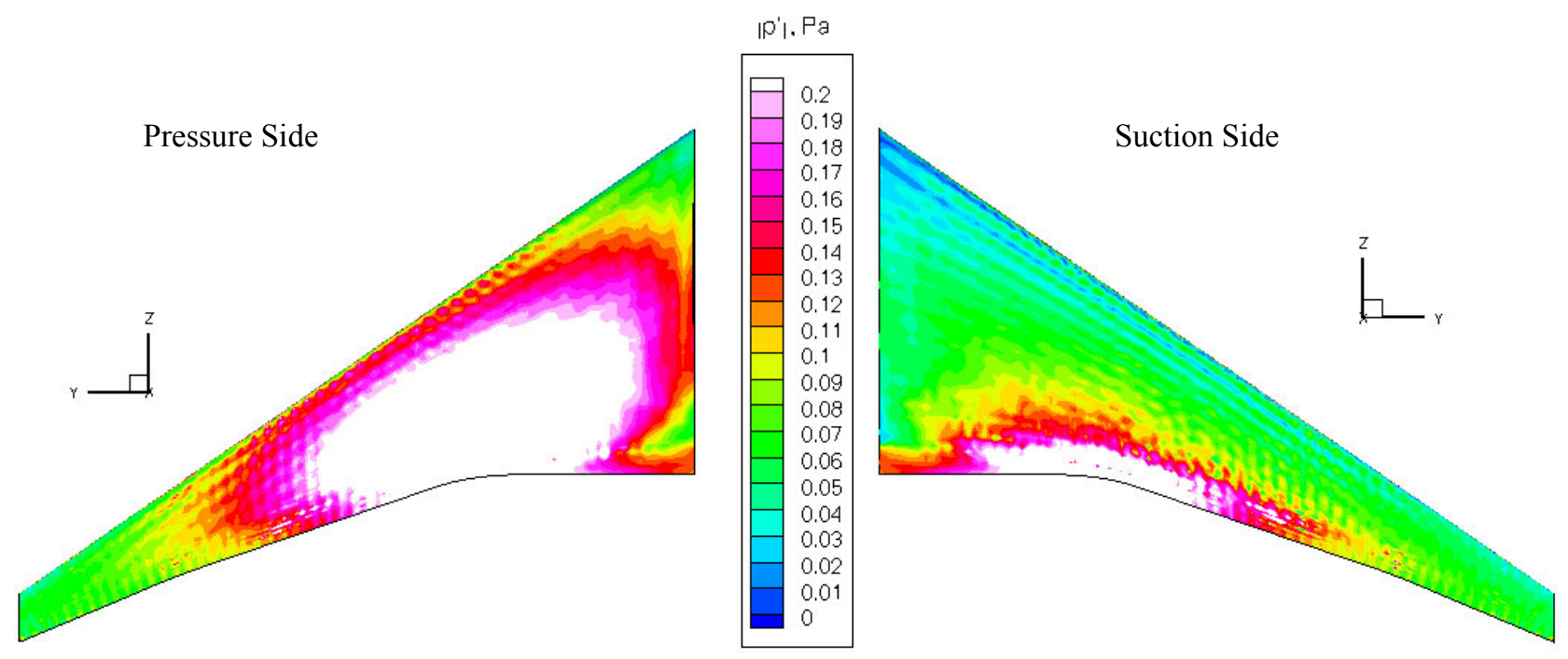

(a) Monopole acoustic source at $190^{\circ}$ from horizontal.
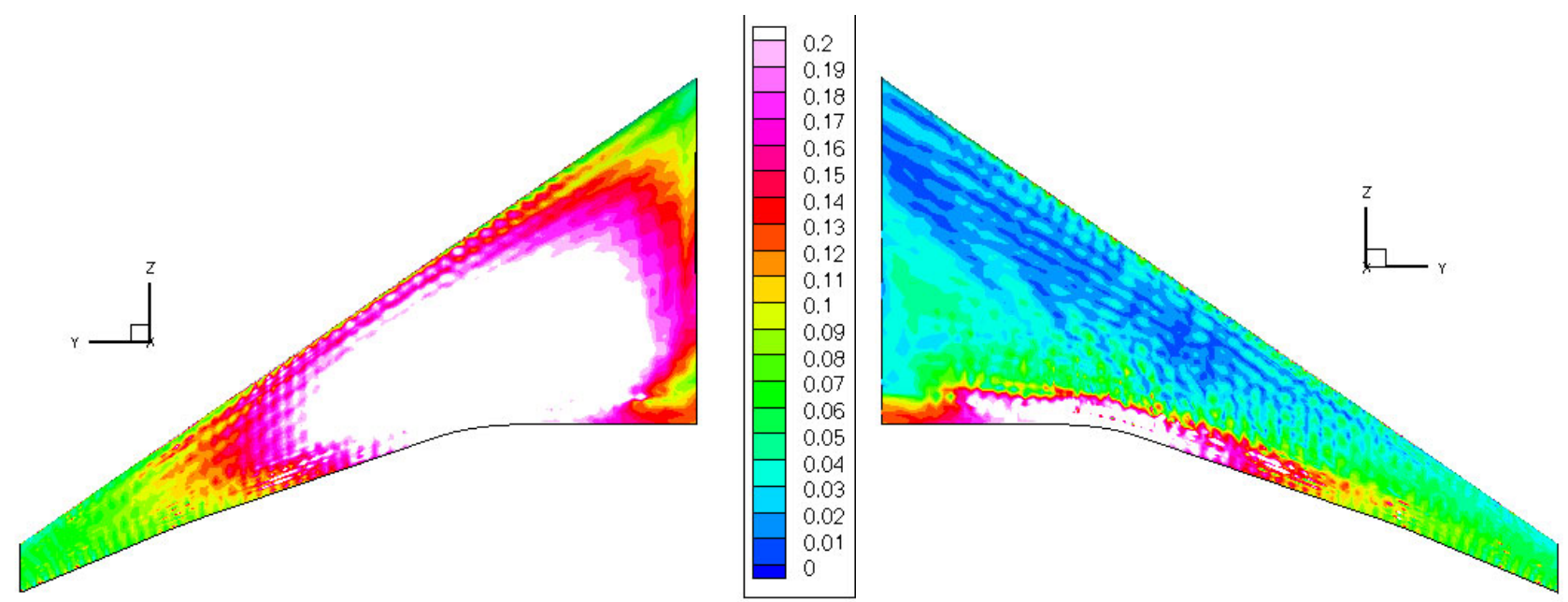

(b) Monopole acoustic source at $200^{\circ}$ from horizontal.
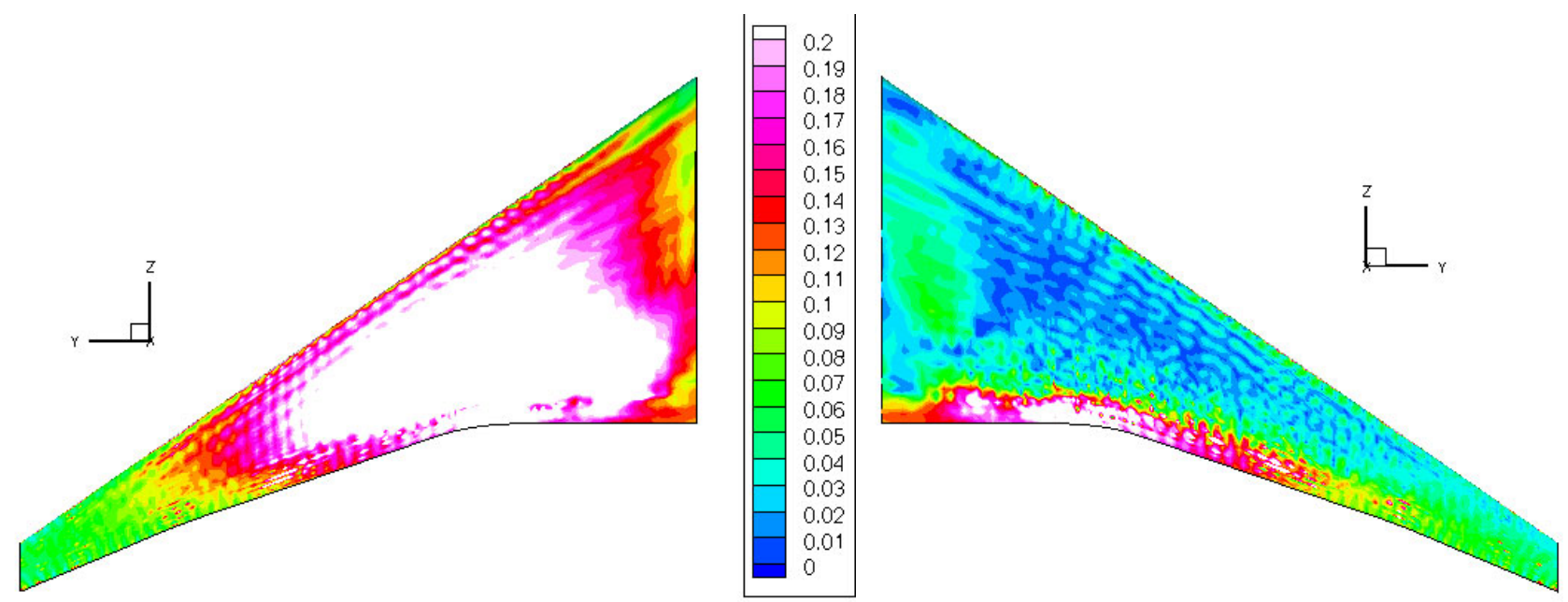

(c) Monopole acoustic source at $210^{\circ}$ from horizontal.

Figure 11- Magnitude of acoustic pressure (Pa) on scaled Boeing 777 wing $(b / 2=2.15 \mathrm{~m})$, $\mathrm{f}=4097 \mathrm{~Hz}, \mathrm{M}=0.2$. 

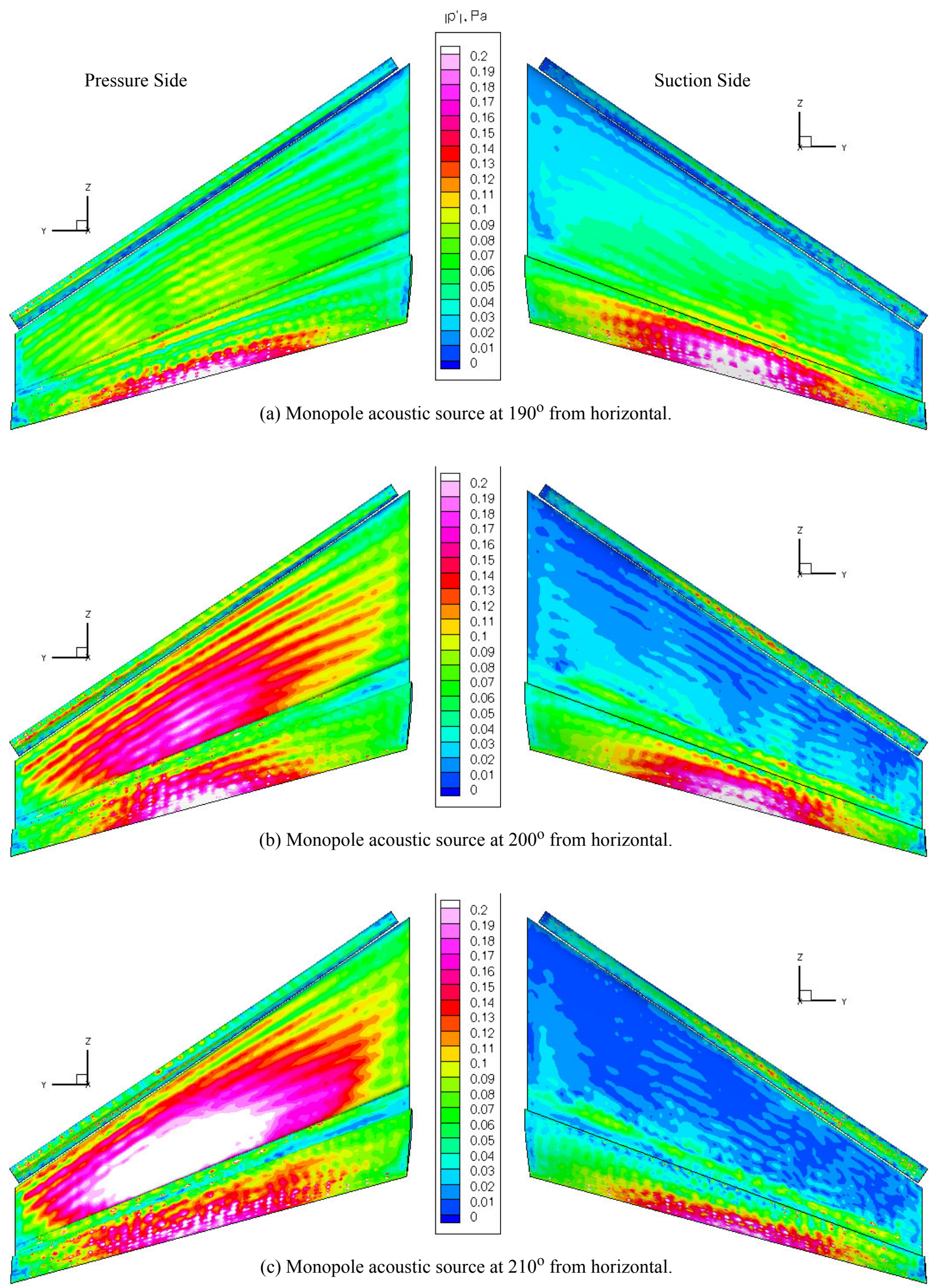

Figure 12- Magnitude of acoustic pressure $(\mathrm{Pa})$ on trap wing high lift system, $\mathrm{f}=3000 \mathrm{~Hz}, \mathrm{M}=0.2$. 

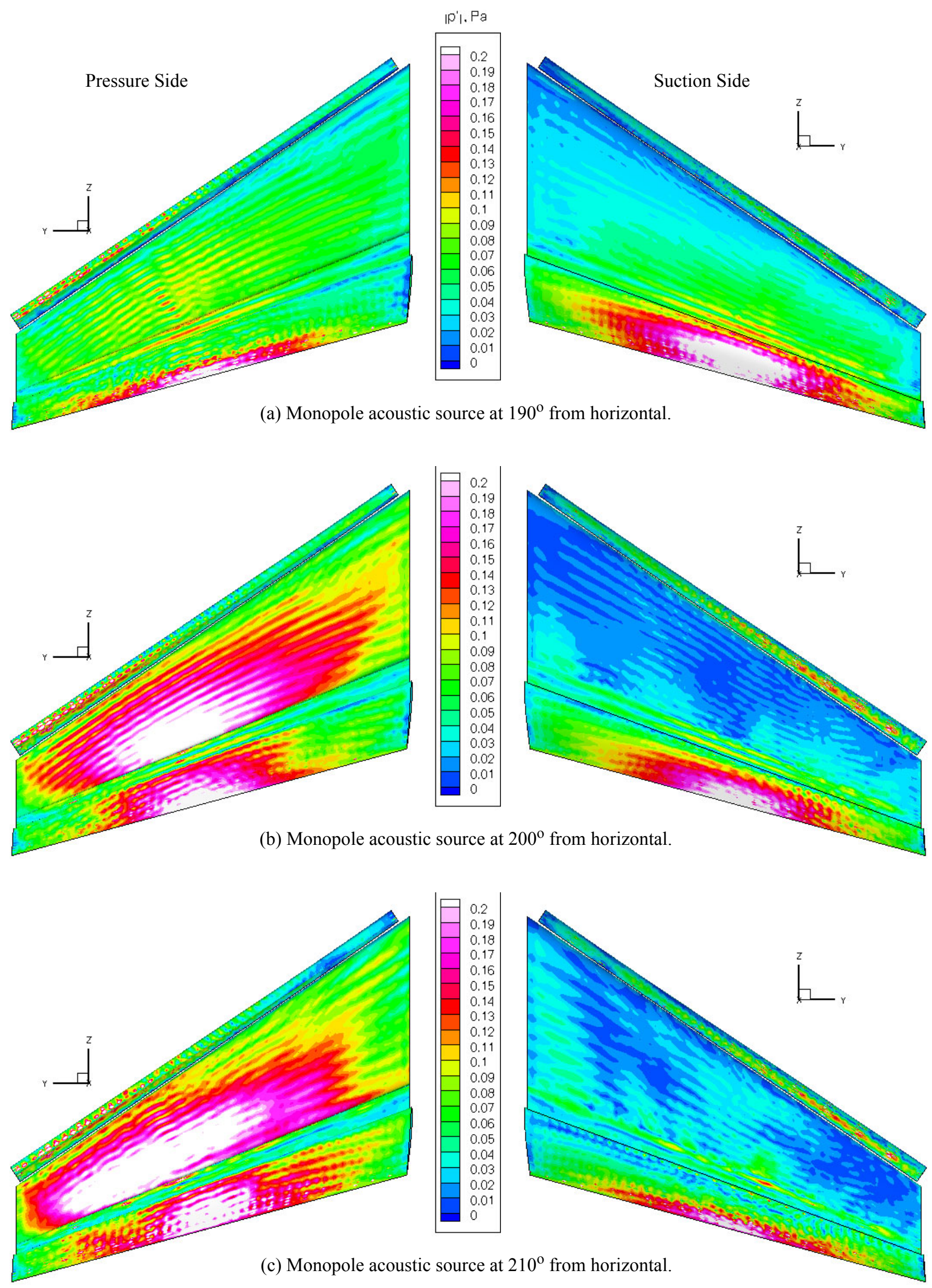

Figure 13- Magnitude of acoustic pressure (Pa) on trap wing high lift system, $\mathrm{f}=4097 \mathrm{~Hz}, \mathrm{M}=0.2$. 